\title{
Fluid Dynamical Limit of the Nonlinear Boltzmann Equation to the Level of the Compressible Euler Equation
}

\section{Takaaki Nishida}

Department of Applied Mathematics and Physics, Kyoto University, Kyoto, 606, Japan

\begin{abstract}
The nonlinear Boltzmann equation for a rarefied gas is investigated in the fluid dynamical limit to the level of compressible Euler equation locally in time, as the mean free path $\varepsilon$ tends to zero. The nonlinear hyperbolic conservation laws obtained as the limit are also the first approximation of the Chapman-Enskog expansion.
\end{abstract}

\section{§1. Introduction}

The dimensionless Boltzmann equation in the kinetic theory of gases can be written for the mass density distribution function $F(t, x, v), t \geqq 0, x \in \mathbb{R}^{3}, v \in \mathbb{R}^{3}$ in the form (cf. [4])

$$
\frac{\partial F}{\partial t}+\Sigma v_{j} \frac{\partial F}{\partial x_{j}}=\frac{1}{\varepsilon} Q(F, F),
$$

where $\varepsilon$ is the mean free path and

$$
Q(F, G)=\frac{1}{2} \int\left(F^{\prime} G_{*}^{\prime}+F_{*}^{\prime} G^{\prime}-F G_{*}-F_{*} G\right) V r d r d \phi d v_{*} .
$$

Here, $V=\left|v-v_{*}\right|, v^{\prime}$ and $v_{*}^{\prime}$ are the velocities after the interaction of the molecules whose velocities were $v, v_{*}$ before the interaction, and $r, \phi$ are the polar coordinate in the impact plane. Also $F_{*}=F\left(t, x, v_{*}\right), F^{\prime}=F\left(t, x, v^{\prime}\right), F_{*}^{\prime}=F\left(t, x, v_{*}^{\prime}\right)$ and $G_{*}, G^{\prime}$, $G_{*}^{\prime}$ are defined analogously. Define the summational invariants

$$
\left\{\Psi_{j}\right\}_{j=1}^{5} \equiv\left\{1, v_{j}(j=1,2,3), v^{2}\right\}
$$

which satisfy

$$
\int \Psi_{j} Q(F, G) d v=0 \quad \text { for } \quad j=1,2, \ldots, 5 \text {. }
$$

The hydrodynamical quantities are defined as follows: The mass density and fluid flow velocity are given by

$$
\begin{aligned}
& \varrho(t, x) \equiv \int F(t, x, v) d v, \\
& u(t, x) \equiv \frac{1}{\varrho} \int v F(t, x, v) d v .
\end{aligned}
$$


The velocity relative to the mean is given by $c=v-u$. The stress tensor and heatflow vector are defined by

$$
\begin{aligned}
P_{i j} & \equiv \int c_{i} c_{j} F(t, x, v) d v=p_{i j}+p \delta_{i j}, \\
q_{i} & \equiv \frac{1}{2} \int c_{i} c^{2} F(t, x, v) d v,
\end{aligned}
$$

where $p \equiv \Sigma P_{k k} / 3$ is the scalar pressure and $p_{i j}$ is defined by (1.7). The internal energy per unit mass is

$$
e \equiv \frac{1}{\varrho} \int \frac{1}{2} c^{2} F(t, x, v) d v
$$

The conservation laws for $\varrho, u, e$ can be written in the form.

$$
\begin{aligned}
& \frac{\partial \varrho}{\partial t}+\Sigma \frac{\partial \varrho u_{j}}{\partial x_{j}}=0 \\
& \frac{\partial \varrho u_{i}}{\partial t}+\Sigma \frac{\partial}{\partial x_{j}}\left(\varrho u_{i} u_{j}+p_{i j}+p \delta_{i j}\right)=0 \\
& \frac{\partial}{\partial t} \varrho\left(e+\frac{1}{2} u^{2}\right)+\Sigma \frac{\partial}{\partial x_{j}}\left\{\varrho u_{j}\left(e+\frac{1}{2} u^{2}\right)\right. \\
& \left.+\Sigma u_{k}\left(p_{k j}+p \delta_{k j}\right)+q_{j}\right\}=0,
\end{aligned}
$$

where the equation of state is that of "the ideal gas", i.e.,

$$
R T \equiv \frac{p}{\varrho}=\frac{2}{3} e .
$$

The system (1.10) is not closed by itself, because for $P_{i j}$ and $q_{i}$ we need the higher momentum of $F$. But if the distribution function $F(t, x, v)$ is locally Maxwellian, i.e.,

$$
F(t, x, v)=\frac{\varrho(t, x)}{(2 \pi R T(t, x))^{3 / 2}} \exp \left(-\frac{(u(t, x)-v)^{2}}{2 R T(t, x)}\right)
$$

then the conservation laws (1.10) can be simplified by $p_{i j}=q_{i}=0$ to

$$
\left.\begin{array}{l}
\frac{\partial \varrho}{\partial t}+\Sigma \frac{\partial \varrho u_{j}}{\partial x_{j}}=0 \\
\frac{\partial \varrho u_{i}}{\partial t}+\Sigma \frac{\partial}{\partial x_{j}}\left(\varrho u_{i} u_{j}+p \delta_{i j}\right)=0 \\
\frac{\partial}{\partial t}\left(\varrho\left(e+\frac{1}{2} u^{2}\right)\right)+\Sigma \frac{\partial}{\partial x_{j}}\left(\varrho u_{j}\left(e+\frac{1}{2} u^{2}\right)+p u_{j}\right)=0 .
\end{array}\right\}
$$


The latter, supplement by (1.11), is now closed and may be considered as the compressible Euler equation derived from the Boltzmann equation. The system of nonlinear hyperbolic conservation laws (1.13) is also obtained as the first approximation of the Chapman-Enskog procedure. The second approximation is the compressible Navier-Stokes equation [1].

Following Grad $[6,8]$ we consider the Boltzmann equation (1.1) for gas molecules with cut-off hard potentials in a neighbourhood of the absolute Maxwellian state:

$$
M(v)=(2 \pi)^{-3 / 2} \exp \left(-v^{2} / 2\right)
$$

Taking initial data

$$
F(0, x, v)=F(x, v),
$$

whose deviation from the absolute Maxwellian state (1.14) is assumed to be small $O(\varepsilon)$, we solve the initial value problem (1.1), for fixed $\varepsilon$, locally in time $[5,7]$ and globally in time $[18,19,14]$ and [17]. The solutions of (1.1) decay to the absolute Maxwellian state as $t \rightarrow+\infty$.

Asymptotic problems of the Boltzmann equation as the mean free path $\varepsilon \rightarrow 0$ and asymptotic relations to the hydrodynamical equations as determined by the Chapman-Enskop expenasion have been considered by Grad [7] for the "semilinear" Boltzmann equations

$$
\frac{\partial f}{\partial t}+\Sigma v_{j} \frac{\partial f}{\partial x_{j}}=\frac{1}{\varepsilon} L f+v \Gamma(f, f)
$$

and

$$
\frac{\partial f}{\partial t}+\Sigma v_{j} \frac{\partial f}{\partial x_{j}}=\frac{1}{\varepsilon} L f+\varepsilon v \Gamma(f, f) .
$$

[Compare them to the full Boltzmann equation (2.14).] He showed as $\varepsilon \rightarrow 0$ that the solution of (1.16) is asymptotic to that of linearized Euler equation in a finite time interval $0 \leqq t \leqq t_{0}<\infty$ and that the solution of (1.17) is asymptotic to that of linearized Navier-Stokes equation in $0<t<t_{0} / \varepsilon$. The asymptotic problems have been also considered by McLennan [10], Ellis-Pinsky [2, 3], and Pinsky [16] for the linearized Boltzmann equation (2.22).

In the present paper we consider the asymptotic problem for the nonlinear Boltzmann equation (1.1) or (2.14) as $\varepsilon \rightarrow 0$, at the level of the nonlinear compressible Euler equation, in the class of analytic functions in a finite time interval $0 \leqq t \leqq t_{0}<\infty$. In $\S 2$ we summarize some results on the linearized Boltzmann equation and define a scale of Banach spaces of analytic functions. In $\S 3$ we solve the initial value problem for the nonlinear Boltzmann equation (1.1) for $\varepsilon \in(0,1]$. It is equivalent to solve a nonlinear integral equation (3.1) by use of the solution of the linearized Boltzmann equation. Then it is shown that there exist the analytic solutions for the nonlinear integral equation (3.1) with small analytic initial data (3.3) in a finite time interval independent of $\varepsilon \in(0,1]$. For this purpose 
we use the spectral theory (cf. $\S 2$ ) of the linearized Boltzmann equation and an abstract nonlinear Cauchy-Kowalewski theorem in a scale of the Banach spaces (cf. $[12,13])$. This abstract theorem is an improved version of Ovsjannikov's [15], where it is used to get the local existence of analytic solutions to the initial value problem for incompressible fluid motion with a free surface (cf. [11]). In $\S 4$ the asymptotic behavior of solutions of the nonlinear Boltzmann equation as the mean free path $\varepsilon$ tends to zero is investigated for a finite time interval. The convergence of the solution $F_{\varepsilon}$ of the Boltzmann equation (1.1) to the locally Maxwellian distribution $F_{0}$ as $\varepsilon \rightarrow 0$ is proved by the uniform estimate and the equicontinuity in $t \in\left(0, t_{0}\right)$ of the solution with respect to $\varepsilon \in(0,1]$ and by the nonnegativity of the solution. Thus it is shown that the Boltzmann equation with small analytic initial data can be approximated locally in time as $\varepsilon \rightarrow 0$ by the compressible Euler equation (1.13). An analogous asymptotic problem was considered in [9] for the Broadwell model of the Boltzmann equation for a simple discrete velocity gas by using $[12,13]$. But the arguments in $\S 4$, especially the treatment of the initial layer, also apply to the Broadwell model and improve the results in [9]. In the Appendix, we prove the nonnegativity of solutions of the Boltzmann equation for non-negative initial data.

\section{§2. Notation and Preliminaries on the Linearized Boltzmann Equation}

Letters $x, v, k \in \mathbb{R}^{3}$ denote independent variables, $L^{p}(\cdot)(\cdot=x, v$, or $k)$ denotes the Lebesgue space of measurable functions whose $p$-the power $(1 \leqq p<+\infty)$ is summable in $\mathbb{R}^{3}$ with the usual norm $|f|_{L^{p(\cdot)}} . H^{l}(x), l \geqq 0$, denotes the Sobolev space of $L^{2}(x)$-functions, the derivatives of which, up to and including order $l$, belong to $L^{2}(x) ; \hat{H}^{l}(k)$ is the Fourier transform of $H^{l}(x)$ with the norm

$$
|\hat{f}|_{\hat{H}^{l}(k)} \equiv\left|\left(1+k^{2}\right)^{l / 2} \hat{f}(k)\right|_{L^{2}(k)} \equiv|f|_{H^{l}(x)} .
$$

$H$ denotes the Lebesgue space of square summable functions in $(x, v) \in \mathbb{R}^{6}$ with the norm

$$
\|f\|=\left(\int|f(x, v)|^{2} d x d v\right)^{1 / 2} .
$$

Let us introduce the partial Fourier transform in $x$ of $f \in H$ as

$$
\hat{f}(k, v)=\frac{1}{(2 \pi)^{3 / 2}} \int e^{-i k \cdot x} f(x, v) d x
$$

and denote $\hat{H}=\{\hat{f} ; f \in H\}$. For $\hat{f} \in \hat{H}$, we define

$$
\|\hat{f}\|^{2}=\int|f(k, v)|^{2} d k d v=\|f\|^{2} .
$$

Definition 2.1. We set $H_{0}=H$ and for each $l>0$, we define $H_{l}$ as the Hilbert subspace of $H$ consisting of all $H^{l}(x)$-valued $L^{2}$-functions of $v \in \mathbb{R}^{3}$ with the norm

$$
\begin{aligned}
\|f\|_{l} & \equiv\left(\int|f(\cdot, v)|_{H^{l}(x)}{ }^{2} d v\right)^{1 / 2} \\
& =\left(\iint\left(1+k^{2}\right)^{l}|\hat{f}(k, v)|^{2} d k d v\right)^{1 / 2} \equiv\|\hat{f}\|_{l}<\infty .
\end{aligned}
$$


Definition 2.2. For each $m, l \geqq 0$, we define $B_{m, l}$ as the subspace of $H$ consisting of all $H^{l}(x)$-valued continuous functions of $v \in R^{3}$ with the property

$$
\left(1+v^{2}\right)^{m / 2}|f(\cdot, v)|_{H^{l}(x)} \rightarrow 0 \quad \text { as } \quad|v| \rightarrow \infty .
$$

The norm for $f \in B_{m, l}$ is defined by

$$
\begin{aligned}
\|f\|_{m, l} & \equiv \sup _{v}\left(1+v^{2}\right)^{m / 2}|f(\cdot, v)|_{H^{l}(x)} \\
& =\sup _{v}\left(1+v^{2}\right)^{m / 2}|\hat{f}(\cdot, v)|_{\hat{H}^{l}(k)}<\infty .
\end{aligned}
$$

It is easy to see that by Fubini's theorem

$$
\|f\|_{l} \leqq C\|f\|_{m, l} \text { for } \quad m>3 / 2, \quad l \geqq 0,
$$

and that by Sobolev's lemma if $f \in B_{m, l}$ for $m, l>3 / 2$, then $f$ is bounded and continuous in $x, v$.

Definition 2.3. For any $l \geqq 0$, we define the scale of Hilbert spaces $S_{l}=\bigcup_{\varrho \geqq 0} H_{l, \varrho}$, where

$$
\begin{aligned}
& H_{l, 0}=H_{l}, \\
& H_{l, \varrho}=\left\{f \in H_{l} ;\|f \mid\|_{l, \varrho} \equiv\left\|e^{|k| \varrho} \hat{f}(k, v)\right\|_{l}<\infty\right\} .
\end{aligned}
$$

Definition 2.4. For any $m, l \geqq 0$, we define the scale of Banach spaces $S_{m, l}$ $=\bigcup_{\varrho \geqq 0} B_{m, l, \varrho}$, where

$$
\begin{aligned}
& B_{m, l, 0}=B_{m, l}, \\
& B_{m, l, \varrho}=\left\{f \in B_{m, l} ;\|f\|_{m, l, \varrho}=\left\|e^{|k| \varrho} \hat{f}(k, v)\right\|_{m, l}<\infty\right.
\end{aligned}
$$

with the property

$$
\left.\lim _{|v| \rightarrow \infty}\left(1+v^{2}\right)^{m / 2}\left|e^{|k| \varrho} \hat{f}(k, v)\right|_{\hat{\boldsymbol{H}}^{l}(k)}=0\right\} .
$$

Then the functions $f(z, v)$ of $H_{l, \varrho}$ or $B_{m, l, \varrho}$ are analytic in $z=x+i y$ for $|y|<\varrho$ and belong to $L^{2}(z)$ for any fixed $y,|y|<\varrho$. The following lemma concerning our scale of analytic functions is fundamental in applications of the abstract nonlinear Cauchy-Kowalewski theorem (cf. Propositions 3.1 and 4.1).

Lemma 2.1. For any $l \geqq 0$, the scale of Hilbert spaces $S_{l}$ has the property:

$$
\left\|\left.|| k\right|^{\sigma} \hat{f}(k, v)\right\|_{l, \varrho^{\prime}} \leqq \frac{1}{\left(\varrho-\varrho^{\prime}\right)^{\sigma}}\|f\|_{l, \varrho}
$$

for any $f \in H_{l, \varrho}$ and any $\varrho^{\prime}<\varrho, 0<\sigma \leqq 1$.

The proof is easily obtained by the definition of the norm $\|\mid\| \|_{l, \varrho}$, if we note that $\begin{aligned}|k|^{\sigma} e^{|k| \varrho^{\prime}} & \leqq|k|^{\sigma}\left(\varrho-\varrho^{\prime}\right)^{\sigma} e^{-|k|\left(\varrho-\varrho^{\prime}\right)} e^{|k| \varrho} /\left(\varrho-\varrho^{\prime}\right)^{\sigma} \\ & \leqq e^{|k| \varrho} /\left(\varrho-\varrho^{\prime}\right)^{\sigma} \text { for any } \varrho^{\prime}<\varrho, 0<\sigma \leqq 1 .\end{aligned}$ 
In order to linearize the Boltzmann equation (1.1) in a neighborhood of the absolute Maxwellian state, we set

$$
F(t, x, v)=M+M^{1 / 2} f(t, x, v) .
$$

If we substitute (2.13) into (1.1) and follow the arguments [6,7] for gas molecules with a cut-off hard potential, we obtain the following equation for $f(t, x, v) t \geqq 0$, $x \in \mathbb{R}^{3}, v \in \mathbb{R}^{3}$ :

$$
\frac{\partial f}{\partial t}+\Sigma v_{j} \frac{\partial f}{\partial x_{j}}=\frac{1}{\varepsilon}(L f+v \Gamma(f, f)) .
$$

Here $L$ is a nonpositive linear operator acting on $v \in \mathbb{R}^{3}$ with the properties that

$$
(L f, f)_{L^{2}(v)} \leqq 0 \text { for } f, L f \in L^{2}(v),
$$

and

$$
L f=0 \quad \text { iff } \quad f \in\left\{\psi_{j}\right\}_{j=1}^{5}=\left\{M^{1 / 2}, v_{j} M^{1 / 2}, v^{2} M^{1 / 2}\right\} .
$$

$L$ can be decomposed as

$$
L=-v(v)+K \text { in } L^{2}(v),
$$

where $v(v)$ is a monotone nondecreasing function in $|v|$ satisfying

$$
0<v_{0} \leqq v(v) \leqq v_{1}(1+|v|), \quad v_{0}, v_{1} \text { positive constants, }
$$

and $K$ is a compact selfadjoint operator on $L^{2}(v)$ with the following smoothing properties :

$$
\begin{aligned}
& \|K f\|_{m, l, \varrho} \leqq \kappa \mid\|f\|_{m-1, l, \varrho} \text { for any } m \geqq 1, \\
& \|\mid K f\|_{0, l, \varrho} \leqq \kappa\|f\|_{l, \varrho}
\end{aligned}
$$

for some constant $\kappa=\kappa(m)<\infty$ and for any $\varrho \geqq 0, l \geqq 0$.

The nonlinear operator

$$
\nu \Gamma(f, g)=\frac{1}{2} \int\left(f^{\prime} g_{*}^{\prime}+f_{*}^{\prime} g^{\prime}-f g_{*}-f_{*} g\right) M\left(v_{*}\right)^{1 / 2} \operatorname{Vr} d r d \phi d v_{*}
$$

acts on $v \in R^{3}$ and is bilinear in $f$ and $g$. It has the following estimate, which will be used in the proof of Propositions 3.1 and 4.1.

Lemma 2.2. Let $f(x, v), g(x, v) \in B_{m, l, \varrho}$ for $\varrho \geqq 0, l>3 / 2, m>5 / 2$. Then $\left(\psi_{j}, v \Gamma(f, g)\right)_{L^{2}(v)}$ $=0, j=1,2, \ldots, 5$, and

$$
\left.\begin{array}{rl}
\|v \Gamma(f, g)\|_{l, \varrho} & \leqq C\|\Gamma(f, g)\| \|_{m, l, \varrho} \\
& \leqq C\|f \mid\|_{m, l, \varrho}\|g\|_{m, l, \varrho} \cdot
\end{array}\right\}
$$

Proof. The first inequality follows easily from (2.8) and (2.18). The second inequality is proved by the argument in [7] and the Hausdorff-Young inequality. 
In fact we have for $l=2$

$$
\begin{aligned}
\|\Gamma(f, g)\| \|_{m, 2, \varrho}= & \sup _{v}\left(1+v^{2}\right)^{m / 2}\left|e^{|k| \varrho}\left(1+k^{2}\right) \Gamma(f, g)^{\wedge}(k, v)\right|_{L^{2}(k)} \\
\leqq & C\left\{\sup \left(1+v^{2}\right)^{m / 2}\left|e^{|k| \varrho} \hat{f}(k, v)\right|_{L^{1}(k)}\right\} \\
& \cdot\left\{\sup \left(1+v^{2}\right)^{m / 2}\left|e^{|k| \varrho}\left(1+k^{2}\right) \hat{g}(k, v)\right|_{L^{2}(k)}\right\} \\
& +\left\{\sup \left(1+v^{2}\right)^{m / 2}\left|e^{|k| \varrho}\left(1+k^{2}\right) \hat{f}\right|_{L^{2}(k)}\right\}\left\{\sup \left(1+v^{2}\right)^{m / 2}\left|e^{|k| \varrho} \hat{g}\right|_{L^{1}(k)}\right\} \\
\leqq & C\left\{\sup \left(1+v^{2}\right)^{m / 2}\left|e^{|k| \varrho}\left(1+k^{2}\right) \hat{f}\right|_{L^{2}(k)}\right\} \\
& \cdot\left\{\sup \left(1+v^{2}\right)^{m / 2}\left|e^{|k| \varrho}\left(1+k^{2}\right) \hat{g}\right|_{L^{2}(k)}\right\} \\
= & C\|f\|_{m, 2, \varrho} \mid\|g\|_{m, 2, \varrho} .
\end{aligned}
$$

This completes the proof of the lemma.

Our aim in the remainder of this section is to summarize some results on the linearized Boltzmann equation

$$
\frac{\partial f}{\partial t}=-\Sigma v_{j} \frac{\partial f}{\partial x_{j}}+\frac{1}{\varepsilon} L f \equiv \frac{1}{\varepsilon} B_{\varepsilon} f .
$$

Consider the two operators

$$
\left.\begin{array}{l}
\frac{1}{\varepsilon} A_{\varepsilon}=-\Sigma v_{j} \frac{\partial f}{\partial x_{j}}-\frac{1}{\varepsilon} v(v), \\
\frac{1}{\varepsilon} B_{\varepsilon}=-\Sigma v_{j} \frac{\partial f}{\partial x_{j}}-\frac{1}{\varepsilon} L f
\end{array}\right\}
$$

with the common domain $D\left(\frac{1}{\varepsilon} A_{\varepsilon}\right)=D\left(\frac{1}{\varepsilon} B_{\varepsilon}\right)$ maximal in $H_{l}, l \geqq 0$. The operator $\frac{1}{\varepsilon} A_{\varepsilon}$ generates a strongly continuous semigroup in $H_{l}$, given explicitly by the formula

$$
e^{\frac{t}{\varepsilon} A_{\varepsilon}} f=e^{-\frac{t}{\varepsilon} v(v)} f\left(x-\frac{t}{\varepsilon} v, v\right)=\frac{1}{(2 \pi)^{3 / 2}} \int e^{i k \cdot x} e^{\frac{t}{\varepsilon} A_{\varepsilon k}} \hat{f}(k, v) d k
$$

where

$$
A_{\varepsilon k}=-i \varepsilon k \cdot v-v(v) .
$$

Since $B_{\varepsilon}=A_{\varepsilon}+K$ and $K$ is a bounded perturbation, the linearized Boltzmann operator $\frac{1}{\varepsilon} B_{\varepsilon}$ also generates a strongly continuous semigroup $\left\{\exp \left(\frac{t}{\varepsilon} B_{\varepsilon}\right)\right\}_{t \geqq 0}$ in $H_{l}$ for any $\varepsilon \in(0,1]$.

We have the following result.

Theorem 2.1. The linear Boltzmann semigroup is represented by

$$
e^{\frac{t}{\varepsilon} B_{\varepsilon}} f=\frac{1}{(2 \pi)^{3 / 2}} \int e^{i k \cdot x} e^{\frac{t}{\varepsilon} B_{\varepsilon k}} \hat{f}(k, v) d k
$$


for $\hat{f}(k, v) \in \hat{H}_{l}$, where for each $k \in \mathbb{R}^{3}$

$$
B_{\varepsilon k}=-i \varepsilon k \cdot v-v(v)+K
$$

is an unbounded linear operator on $L^{2}(v)$ with domain $D\left(B_{\varepsilon k}\right)=\left\{f \in L^{2}(v)\right.$, $\left.B_{\varepsilon k} f \in L^{2}(v)\right\}, B_{\varepsilon k}$ generates a strongly continuous contraction semigroup on $L^{2}(v)$ such that for any $t \geqq 0, f \in L^{2}(v)$,

$$
\left|e^{\frac{t}{\varepsilon} B_{\varepsilon k}} f\right|_{L^{2}(v)} \leqq|f|_{L^{2}(v)}
$$

Furthermore there exist $\delta, \beta_{1}, \beta_{2}>0$ such that the following Eqs. $i$ ) and ii) are valid for any $f \in D\left(B_{\varepsilon k}\right)$.

i) For any $k,|\varepsilon k|<\delta$

$$
\begin{aligned}
e^{\frac{t}{\varepsilon} B_{\varepsilon k}} f= & \sum_{j=1}^{5} e^{\frac{t}{\varepsilon} \alpha_{j}(\varepsilon k)}\left(e_{j}(-\varepsilon k), f\right)_{L^{2}(v)} e_{j}(\varepsilon k) \\
& +e^{\frac{t}{\varepsilon} A_{\varepsilon k}} f+e^{-\frac{t}{\varepsilon} \beta_{1}} Z_{1}(\varepsilon k, t / \varepsilon) f,
\end{aligned}
$$

where $\alpha_{j}, e_{j}$ are the eigenvalues and the corresponding eigenfunctions of $B_{\varepsilon k}$. They have the expansions

$$
\left.\begin{array}{l}
\alpha_{j}(\varepsilon k)=\sum_{n=1}^{3} a_{j, n}(i \varepsilon|k|)^{n}+O\left(|\varepsilon k|^{4}\right) \\
e_{j}(\varepsilon k)=\sum_{n=0}^{3} e_{j, n}(k /|k|)(i \varepsilon|k|)^{n}+O\left(|\varepsilon k|^{4}\right),
\end{array}\right\}
$$

where $a_{j, n}$ are constants, $a_{j, 2}>0$ and

$$
\left(e_{j}(-\varepsilon k), e_{n}(\varepsilon k)\right)_{L^{2}(v)}=\delta_{j, n}, \quad j, n=1, \ldots, 5 \text {. }
$$

ii) For any $k,|\varepsilon k|>\delta$

$$
e^{\frac{t}{\varepsilon} B_{\varepsilon k}} f=e^{\frac{t}{\varepsilon} A_{\varepsilon k}} f+e^{-\frac{t}{\varepsilon} \beta_{2}} Z_{2}(\varepsilon k, t / \varepsilon) f,
$$

where

$$
\begin{aligned}
Z_{j}(\varepsilon k, t / \varepsilon) f & =\lim _{\gamma \rightarrow \infty} \frac{1}{2 \pi} \int_{-i \gamma}^{i \gamma} e^{i \frac{t}{\varepsilon} \gamma} Z\left(-\beta_{j}+\mathrm{i} \gamma, \varepsilon k\right) f d \gamma \\
Z(\lambda, \varepsilon k) & =\left(\lambda-A_{\varepsilon k}\right)^{-1}\left(I-K\left(\lambda-A_{\varepsilon k}\right)^{-1}\right)^{-1} K\left(\lambda-A_{\varepsilon k}\right)^{-1}
\end{aligned}
$$

and

$$
\left|Z_{j}(\varepsilon k, t / \varepsilon) f\right|_{L^{2}(v)} \leqq C|f|_{L^{2}(v)},
$$

$C$ being independent of $\varepsilon, k, t \geqq 0$.

Proof. See Ellis-Pinsky [2], Ukai [19], and Nishida-Imai [14]. 
Proposition 2.1. The linearized Boltzmann operator $\frac{1}{\varepsilon} B_{\varepsilon}$ also generates a strongly continuous semigroup in $B_{m, l, \varrho}(m>3 / 2, l \geqq 0, \varrho \geqq 0)$ and satisfies a uniform estimate

$$
\left\|e^{\frac{t}{\varepsilon} B_{\varepsilon}} f\right\|_{m, l, \varrho} \leqq C\|\| f \|_{m, l, \varrho},
$$

for any $m>3 / 2, l \geqq 0, \varrho \geqq 0, C$ being independent of $\varepsilon \in(0,1]$.

Proof. The first part comes from the property (2.11) of $B_{m, l, \varrho}$; cf. Ukai-Nishida [20] and also Shizuta [17]. It follows from (2.26), (2.28), and the Plancherel equality that for any $f \in H_{l, \varrho}, l \geqq 0, \varrho \geqq 0$, any $t \geqq 0$, and for any $\varepsilon \in(0,1]$

$$
\begin{aligned}
\left\|e^{\frac{t}{\varepsilon} B_{\varepsilon}} f\right\|_{l, \varrho} & =\int\left|e^{\frac{t}{\varepsilon} B_{\varepsilon k}} e^{|k| \varrho}\left(1+k^{2}\right)^{l / 2} \hat{f}(k, v)\right|_{L^{2}(v)}^{2} d k \\
& \leqq\|f \mid\|_{l, \varrho} .
\end{aligned}
$$

In order to get the estimate in \|\|$\|_{m, l, \varrho}$ we use the relation

$$
f_{0}(t)=e^{\frac{t}{\varepsilon} B_{\varepsilon}} f=e^{\frac{t}{\varepsilon} A_{\varepsilon}} f+\int_{0}^{t} e^{\frac{t-s}{\varepsilon} A_{\varepsilon}} \frac{K}{\varepsilon} f_{0}(s) d s .
$$

It is easy to see that for any $\varrho \geqq 0, l \geqq 0, m \geqq 0$

$$
\left\|e^{\frac{t}{\varepsilon} A_{\varepsilon}} f\right\|_{m, l, \varrho} \leqq e^{-\frac{t}{\varepsilon} v_{0}}\|f\|_{m, l, \varrho} .
$$

It follows from (2.19), (2.36), and (2.37) that

$$
\left\|e^{\frac{t}{\varepsilon} B_{\varepsilon}} f\right\|_{j, l, \varrho} \leqq C\left\{\|f\|_{l, \varrho}+\sum_{i=0}^{j}\|f\|_{i, l, \varrho}\right\}
$$

for any $f \in B_{j, l, \varrho} \cap H_{l, \varrho}(j=0,1, \ldots, m$ inductively) and $\varrho \geqq 0$. Thus if $m>3 / 2$, then (2.8) gives (2.34).

This completes the proof.

In addition to Theorem 2.1 and the uniform bound (2.34) for $\varepsilon \in(0,1]$, we need the Hölder continuity in $t$, uniformly for $\varepsilon \in(0,1]$. of solutions of the linearized Boltzmann equation. It will be formulated in Proposition 2.2 after the following two auxiliary lemmas.

Lemma 2.3. Let $f \in B_{m, l, \varrho}(\varrho \geqq 0, l, m>0)$ and consider $\exp \left(\frac{t}{\varepsilon} A_{\varepsilon}\right) f$ in $t \geqq 0, \varepsilon \in(0,1]$.

Then we have for $0<\sigma<\min (1, l, m)$

$$
\left\|e^{\frac{t}{\varepsilon} A_{\varepsilon}} f-e^{\frac{s}{\varepsilon} A_{\varepsilon}} f\right\|_{m-\sigma, l-\sigma, \varrho} \leqq C\left(\frac{t-s}{s}\right)^{\sigma}\|f\|_{m, l, \varrho}
$$

for $0<s<t$, where $C$ is independent of $\varepsilon \in(0,1]$.

Proof. Remembering that

$$
\left(e^{\frac{t}{\varepsilon} A_{\varepsilon}} f\right)=e^{-\frac{t}{\varepsilon}(v(v)+i \varepsilon k \cdot v)} \hat{f}(k, v)
$$


we have

$$
\begin{aligned}
& \left\|e^{\frac{t}{\varepsilon} A_{\varepsilon}} f-e^{\frac{s}{\varepsilon} A_{\varepsilon}} f\right\|_{m-\sigma, l-\sigma, \varrho}=\sup _{v}\left(1+v^{2}\right)^{\frac{m-\sigma}{2}} \|\left(e^{-\frac{t}{\varepsilon}(v(v)+i \varepsilon k \cdot v)}-e^{-\frac{s}{\varepsilon}(v(v)+i \varepsilon k \cdot v)}\right) \\
& \left.\cdot e^{|k| \varrho}\left(1+k^{2}\right)^{\frac{l-\sigma}{2}} \hat{f}(k, v)\right|_{L^{2}(k)} \\
& \leqq \sup \left(1+v^{2}\right)^{\frac{m-\sigma}{2}}\left(1-e^{-\frac{t-s}{\varepsilon}(v(v)+i \varepsilon k \cdot v)}\right) e^{-\frac{s}{\varepsilon}(v(v)+i \varepsilon k \cdot v)} \\
& \left.\cdot e^{|k| \varrho}\left(1+k^{2}\right)^{\frac{l-\sigma}{2}} \hat{f}\right|_{L^{2}(k)} \\
& \leqq C \sup \left(1+v^{2}\right)^{\frac{m-\sigma}{2}} \mid\left\{\left(\frac{t-s}{\varepsilon} v(v)\right)^{\sigma}+e^{-\frac{t-s}{\varepsilon} v(v)}|(t-s) k \cdot v|^{\sigma}\right\} \\
& \left.\cdot e^{-\frac{s}{\varepsilon} v(v)} e^{|k| \varrho}\left(1+k^{2}\right)^{\frac{l-\sigma}{2}} \hat{f}\right|_{L^{2}(k)} \\
& \leqq C \sup \left(1+v^{2}\right)^{\frac{m-\sigma}{2}}\left(\frac{t-s}{s}\right)^{\sigma} \mid\left(\frac{s}{\varepsilon} v(v)\right)^{\sigma} e^{-\frac{s}{\varepsilon} v(v)}\left(1+\frac{|k \cdot v|^{\sigma}}{v(v)}\right) \\
& \left.\cdot e^{|k| \varrho}\left(1+k^{2}\right)^{\frac{l-\sigma}{2}} \hat{f}\right|_{L^{2}(k)} \\
& \leqq C\left(\frac{t-s}{s}\right)^{\sigma} \sup \left(1+v^{2}\right)^{m / 2}\left|\left(1+k^{2}\right)^{l / 2} e^{|k| \varrho} \hat{f}\right|_{L^{2}(k)} \\
& =C\left(\frac{t-s}{s}\right)^{\sigma}\|f\|_{m, l, \varrho} \text {. }
\end{aligned}
$$

This completes the proof.

Lemma 2.4. Let $g(t) \in B_{m, l, \varrho}$ for each $t \in[0, T]$ and for some $\varrho \geqq 0, l, m>0$. Put $\sup _{0 \leqq t \leqq T}\|g(t)\|_{m, l, \varrho}=C_{0}$ and assume

$$
\|g(t)-g(s)\| \|_{m-\sigma, l-\sigma, \varrho} \leqq C_{1}\left(\frac{t-s}{s}\right)^{\sigma} \text { for } \quad 0<s<t \leqq T
$$

and some $\sigma \in(0, \min (1, l, m))$.

Then for $0<s<t \leqq T, T>0$ arbitrary,

$$
f(t)=\int_{0}^{t} e^{\frac{t-s}{\varepsilon} A_{\varepsilon}} \frac{v(v)}{\varepsilon} g(s) d s
$$

satisfies the estimate

$$
\left.\begin{array}{l}
\|f(t)\|_{m, l, \varrho} \leqq C_{0}, \\
\|f(t)-f(s)\|_{m-\sigma, l-\sigma, \varrho} \leqq C\left(C_{0}+C_{1}\right)\left(\frac{t-s}{s}\right)^{\sigma}
\end{array}\right\}
$$


where $C$ is independent of $\varepsilon \in(0,1]$.

Proof. We have

$$
\begin{aligned}
& \|\| f(t)-f(s) \|_{m-\sigma, l-\sigma, \varrho} \\
& =\left\|\int_{0}^{t} e^{\frac{\tau}{\varepsilon} A_{\varepsilon} \nu} \frac{\nu}{\varepsilon} g(t-\tau) d \tau-\int_{0}^{s} e^{\frac{\tau}{\varepsilon} A_{\varepsilon}} \frac{\nu}{\varepsilon} g(s-\tau) d \tau \mid\right\|_{m-\sigma, l-\sigma, \varrho} \\
& =\left\|\int_{0}^{s} e^{\frac{\tau}{\varepsilon} A_{\varepsilon}} \frac{v}{\varepsilon}(g(t-\tau)-g(s-\tau)) d \tau+\int_{s}^{t} e^{\frac{\tau}{\varepsilon}} A_{\varepsilon} \frac{v}{\varepsilon} g(t-\tau) d \tau\right\| \| \\
& \leqq \int_{0}^{s / 2}\|\| e^{-\frac{\tau}{\varepsilon}(v(v)+i \varepsilon k \cdot v)} \frac{v}{\varepsilon}(\hat{g}(t-\tau)-\hat{g}(s-\tau))\|\| d \tau \\
& +\int_{s / 2}^{s}\|W\| d \tau+\int_{s}^{t}\left\|e^{-\frac{\tau}{\varepsilon}(v(v)+i \varepsilon k \cdot v)} \frac{\nu}{\varepsilon} \hat{g}(t-\tau)\right\| d \tau \\
& \leqq C_{1} \sup _{v} \int_{0}^{s / 2}\left(\frac{t-s}{s-s / 2}\right)^{\sigma} e^{-\frac{\tau}{\varepsilon} \nu} \frac{\nu}{\varepsilon} d \tau \\
& +C_{1}(t-s)^{\sigma} \sup _{v} \int_{s / 2}^{s} \frac{d \tau}{(s-\tau)^{\sigma}}\left(e^{-\frac{\tau}{\varepsilon} \nu} \frac{v}{\varepsilon} \tau\right) \frac{1}{s / 2}+C_{0} \sup _{v} \int_{s}^{t} e^{-\frac{\tau}{\varepsilon} v} \frac{\nu}{\varepsilon} d \tau \\
& \leqq C\left(C_{0}+C_{1}\right)\left(\frac{t-s}{s}\right)^{\sigma} \text {. }
\end{aligned}
$$

The term $W$ in the second integral after the first inequality denotes the same expression which appears in the first integral after this inequality. This completes the proof.

Proposition 2.2. Let $f \in B_{m, l, \varrho}$ for some $\varrho \geqq 0, l>0, m>3 / 2$ and set $f_{0}(t)=e^{\frac{t}{\varepsilon} B_{\varepsilon}} f$. Then we have for $0<\sigma<\min (1 / 2, l, m-3 / 2)$

$$
\begin{aligned}
& \left\|f_{0}(t)-f_{0}(s)\right\|_{l-\sigma, \varrho} \leqq C\left(\frac{t-s}{s}\right)^{\sigma}\|f \mid\|_{m, l, \varrho}, \\
& \|\| f_{0}(t)-f_{0}(s)\left\|_{m-\sigma, l-\sigma, \varrho} \leqq C\left(\frac{t-s}{s}\right)^{\sigma}\right\| f \|_{m, l, \varrho},
\end{aligned}
$$

where $C$ is independent of $\varepsilon \in(0,1]$.

$N . B$. Here we may note that the singularity $1 / t^{\sigma}$ of the Hölder coefficients near $t=0$ corresponds to the initial layer of the rarefied gas motion described by the Boltzmann equation.

Proof. We recall the representation of $f_{0}(t)$ in Theorem 2.1:

$$
\begin{aligned}
f_{0}(t) & =\frac{1}{(2 \pi)^{3 / 2}} \int e^{i k \cdot x} e^{\frac{t}{\varepsilon} B_{\varepsilon k}} \hat{f}(k, v) d k \\
& =\int_{|\varepsilon k|<\delta}+\int_{|\varepsilon k|>\delta} \equiv I_{1}(t)+I_{2}(t) .
\end{aligned}
$$


$I_{2}$ has the representation

$$
\begin{aligned}
I_{2}(t) & =\frac{1}{(2 \pi)^{3 / 2}} \int_{|\varepsilon k|>\delta} e^{i k \cdot x}\left\{e^{\frac{t}{\varepsilon} A_{\varepsilon k}} \hat{f}(k, v)+\lim _{\gamma \rightarrow \infty} \frac{1}{2 \pi i} \int_{-\beta_{2}-i \gamma}^{-\beta_{2}+i \gamma} e^{\frac{t}{\varepsilon} \lambda} Z(\lambda, \varepsilon k) \hat{f}(k, v) d \lambda\right\} d k \\
& \equiv I_{3}(t)+I_{4}(t) .
\end{aligned}
$$

$I_{3}$ can be treated in the same way as in Lemma 2.3:

$$
\begin{aligned}
\left\|I_{3}(t)-I_{3}(s)\right\|_{l-\sigma, \varrho} & \leqq C\left\|I_{3}(t)-I_{3}(s)\right\|_{m-\sigma, l-\sigma, \varrho} \\
& \leqq C\left(\frac{t-s}{s}\right)^{\sigma}\|f\|_{m, l, \varrho} \text { for } 0<\sigma<\min (1, l, m-3 / 2) .
\end{aligned}
$$

To estimate $I_{4}$ we consider the integrand

$$
\begin{aligned}
\lim _{\gamma \rightarrow \infty} & \frac{1}{2 \pi i} \int_{-\beta_{2}-i \gamma}^{-\beta_{2}+i \gamma}\left(e^{\frac{t}{\varepsilon} \lambda}-e^{\frac{s}{\varepsilon}} \lambda\right) Z(\lambda, \varepsilon k) \hat{f}(k, v) d \lambda \\
= & \left(e^{-\beta_{2} \frac{t-s}{\varepsilon}}-1\right) e^{-\beta_{2} \frac{s}{\varepsilon}} \lim _{\gamma \rightarrow \infty} \frac{1}{2 \pi} \int_{-\gamma}^{\gamma} e^{i \frac{t \gamma}{\varepsilon}} Z\left(-\beta_{2}-i \gamma, \varepsilon k\right) \hat{f} d \gamma \\
& +e^{-\beta_{2} \frac{s}{\varepsilon}} \lim _{\gamma \rightarrow \infty} \frac{1}{2 \pi} \int_{-\gamma}^{\gamma}\left(e^{i \frac{t-s}{\varepsilon} \gamma}-1\right) e^{i \frac{s \gamma}{\varepsilon}} Z\left(-\beta_{2}-i \gamma, \varepsilon k\right) \hat{f} d \gamma \\
\equiv & I_{5}+I_{6} .
\end{aligned}
$$

The estimate

$$
\left|I_{5}\right|_{L^{2}(v)} \leqq C\left(\frac{t-s}{s}\right)^{\sigma}|\hat{f}(k, v)|_{L^{2}(v)}
$$

is given by (2.32) and (2.33). $I_{6}$ can be treated as follows:

$$
\begin{aligned}
I_{6}= & e^{-\beta_{2} \frac{s}{\varepsilon}}\left(\frac{t-s}{\varepsilon}\right)^{\sigma} \lim _{\gamma \rightarrow \infty} \frac{1}{2 \pi} \int_{-\gamma}^{\gamma}|\gamma|^{\sigma} \frac{\left(e^{i \frac{t-s}{\varepsilon} \gamma}-1\right)}{\left(\frac{t-s}{\varepsilon}|\gamma|\right)^{\sigma}} e^{i \frac{s \gamma}{\varepsilon} Z\left(-\beta_{2}-i \gamma, \varepsilon k\right) \hat{f} d \gamma} \\
& \equiv C\left(\frac{t-s}{s}\right)^{\sigma} \bar{Z}_{2}\left(\beta_{2}, \varepsilon k, s / \varepsilon, t / \varepsilon\right) \hat{f} .
\end{aligned}
$$

We claim that $\bar{Z}_{2}\left(\beta_{2}, \varepsilon k, s / \varepsilon, t / \varepsilon\right)$ is weakly absolutely convergent for

$$
\left(1+|\varepsilon k|^{2}\right)^{\sigma / 2}\left(1+v^{2}\right)^{\sigma / 2} \hat{f}(k, v) \in L^{2}(v),
$$

and

$$
\left|\bar{Z}_{2} \hat{f}\right|_{L^{2}(v)} \leqq C\left(1+|\varepsilon k|^{2}\right)^{\sigma / 2}\left|\left(1+v^{2}\right)^{\sigma / 2} \hat{f}\right|_{L^{2}(v)}
$$


where $C$ is independent of $k, 0<s<t$ and $\varepsilon \in(0,1]$. In fact

$$
\begin{aligned}
& \lim _{\gamma \rightarrow \infty} \frac{1}{2 \pi} \int_{-\gamma}^{\gamma}|\gamma|^{\sigma} \mid \frac{\left(e^{i \frac{t-s}{\varepsilon} \gamma}-1\right)}{\left(\frac{t-s}{\varepsilon}|\gamma|\right)^{\sigma}} e^{i \frac{s \gamma}{\varepsilon}}\left(\left(I-K\left(-\beta_{2}-i \gamma-A_{\varepsilon k}\right)^{-1}\right)^{-1}\right. \\
& \left.\cdot K\left(-\beta_{2}-i \gamma-A_{\varepsilon k}\right)^{-1} \hat{f}(k, v),\left(-\beta_{2}+i \gamma-A_{\varepsilon k}^{*}\right)^{-1} \hat{g}(k, v)\right)_{L^{2}(v)} d \gamma \\
& \leqq \lim \frac{C}{2 \pi}\left\{\int_{-\gamma}^{\gamma} \frac{\left(|\gamma|^{\sigma}|\hat{f}(k, v)|\right)^{2}}{\left(v(v)-\beta_{2}\right)^{2}+(\gamma+\varepsilon k \cdot v)^{2}} d v d \gamma\right. \\
& \left.\cdot \int_{-\gamma}^{\gamma} \frac{\left(|\gamma|^{\sigma}|\hat{g}(k, v)|\right)^{2}}{\left(v(v)-\beta_{2}\right)^{2}+(\gamma+\varepsilon k \cdot v)^{2}} d v d \gamma\right\}^{1 / 2} \\
& \leqq \lim \frac{C}{2 \pi}\left\{\int|\hat{f}|^{2} d v \int_{-\gamma}^{\gamma} \frac{|\varepsilon k \cdot v|^{2 \sigma}+|\gamma+\varepsilon k \cdot v|^{2 \sigma}}{\left(v_{0}-\beta_{2}\right)^{2}+(\gamma+\varepsilon k \cdot v)^{2}} d \gamma\right. \\
& \left.\cdot \int|\hat{g}|^{2} d v \int_{-\gamma}^{\gamma} \frac{|\varepsilon k \cdot v|^{2 \sigma}+|\gamma+\varepsilon k \cdot v|^{2 \sigma}}{\left(v_{0}-\beta_{2}\right)^{2}+(\gamma+\varepsilon k \cdot v)^{2}} d \gamma\right\}^{1 / 2} \\
& \leqq \lim C\left\{\left(|\varepsilon k|^{2 \sigma} \int|\hat{f}|^{2}|v|^{2 \sigma} d v \int_{-\gamma}^{\gamma} \frac{d \gamma}{\left(v_{0}-\beta_{2}\right)^{2}+\gamma^{2}}\right.\right. \\
& \left.+\int|\hat{f}|^{2} d v \int_{-\gamma}^{\gamma} \frac{|\gamma|^{2 \sigma}}{\left(v_{0}-\beta_{2}\right)^{2}+\gamma^{2}} d \gamma\right)\left(|\varepsilon k|^{2 \sigma} \int|\hat{g}|^{2}|v|^{2 \sigma} d v\right. \\
& \left.\left.\cdot \int_{-\gamma}^{\gamma} \frac{d \gamma}{\left(v_{0}-\beta_{2}\right)^{2}+\gamma^{2}}+\int|\hat{g}|^{2} d v \int_{-\gamma}^{\gamma} \frac{|\gamma|^{2 \sigma} d \gamma}{\left(v_{0}-\beta_{2}\right)^{2}+\gamma^{2}}\right)\right\}^{1 / 2} \\
& \leqq C\left|\left(1+|\varepsilon k|^{2}\right)^{\sigma / 2}\left(1+v^{2}\right)^{\sigma / 2} \hat{f}(k, v)\right|_{L^{2}(v)} \cdot\left|\left(1+|\varepsilon k|^{2}\right)^{\sigma / 2}\left(1+v^{2}\right)^{\sigma / 2} \hat{g}(k, v)\right|_{L^{2}(v)}
\end{aligned}
$$

for $0<\sigma<1 / 2$. Thus for $\varepsilon \in(0,1]$

$$
\begin{aligned}
\left\|I_{4}(t)-I_{4}(s)\right\|_{l-\sigma, \varrho} & \leqq C\left(\frac{t-s}{s}\right)^{\sigma} \mid\left(1+|\varepsilon k|^{2}\right)^{\sigma / 2}\left(1+k^{2}\right)^{\frac{l-\sigma}{2}} e^{|k| e \mid} \\
& \cdot\left|\left(1+v^{2}\right)^{\sigma / 2} \hat{f}(k, v)\right|_{L^{2}(v)} \mid L^{2}(k) \\
& \leqq C\left(\frac{t-s}{s}\right)^{\sigma}\left\|\left(1+k^{2}\right)^{l / 2} e^{|k| e}\left(1+v^{2}\right)^{\sigma / 2} \hat{f}(k, v)\right\|_{L^{2}(k, v)} \\
& \leqq C\left(\frac{t-s}{s}\right)^{\sigma}\|\hat{f}(k, v)\|_{m, l, \varrho} .
\end{aligned}
$$

To prove the Hölder continuity of $I_{1}(t)$, we can treat it by (2.29) in the same way as $I_{3}(t)$ and $I_{4}(t)$ except for the term

$$
I_{7}(t)=\frac{1}{(2 \pi)^{3 / 2}} \int_{|\varepsilon k|<\delta} e^{i k \cdot x} \sum_{j=1}^{5} e^{e^{\frac{t}{\varepsilon}} \alpha_{j}(\varepsilon k)}\left(e_{j}(-\varepsilon k), \hat{f}\right)_{L^{2}(v)} e_{j}(\varepsilon k) d k .
$$


But this can be treated as follows:

$$
\begin{aligned}
\| & \left|I_{7}(t)-I_{7}(s)\right| \|_{l-\sigma, \varrho} \\
\leqq & \sum_{j=1}^{5}\left(\int_{|\varepsilon k|<\delta}\left|\left(e^{\frac{t}{\varepsilon} \alpha_{j}(\varepsilon k)}-e^{\frac{s}{\varepsilon} \alpha_{j}(\varepsilon k)}\right)\left(1+k^{2}\right)^{\frac{l-\sigma}{2}} \cdot e^{|k| \varrho} \hat{f}\right|_{L^{2}(v)}^{2} d k\right)^{1 / 2} \\
\leqq & \sum_{j=1}^{5}\left(\int_{|\varepsilon k|<\delta} \mid e^{-\frac{s}{\varepsilon} \alpha_{j}(\varepsilon k)}\right. \\
& \cdot\left\{\left(\frac{t-S}{\varepsilon} \alpha_{j}^{\prime \prime}(\theta \varepsilon k)(\varepsilon k)^{2} / 2\right)^{\sigma}+e^{-\frac{t-s}{\varepsilon} \alpha_{j}^{\prime \prime}(\theta \varepsilon k)(\varepsilon k)^{2} / 2}\left|(t-s) \alpha_{j 1} k\right|^{\sigma}\right\} \\
& \left.\left.\cdot\left(1+k^{2}\right)^{\frac{l-\sigma}{2}} e^{|k| \varrho} \hat{f}\right|_{L^{2}(v)} d k\right)^{1 / 2} \leqq C(t-s)^{\sigma} \mid\|f\|_{l, \varrho} .
\end{aligned}
$$

Since we have shown (2.40), we can use the expression (2.36) to obtain the desired estimate (2.41), where the first term is estimated as in Lemma 2.3 and the second term is estimated by (2.40) and the smoothing properties of $K(2.19)$. In fact

$$
\begin{aligned}
& \left\|\int_{0}^{t} e^{\frac{t-\tau}{\varepsilon} A_{\varepsilon}} \frac{K}{\varepsilon} f_{0}(\tau) d \tau-\int_{0}^{s} e^{\frac{s-\tau}{\varepsilon} A_{\varepsilon}} \frac{K}{\varepsilon} f_{0}(\tau) d \tau\right\|_{0, l-\sigma, \varrho} \\
& =\left\|\int_{s}^{t} e^{\frac{t-\tau}{\varepsilon} A_{\varepsilon}} \frac{K}{\varepsilon} f_{0}(\tau) d \tau\right\| \|_{0, l-\sigma, \varrho} \\
& +\left\|\int_{0}^{s} e^{\frac{\tau}{\varepsilon}} A_{\varepsilon} \frac{K}{\varepsilon}\left(f_{0}(t-\tau)-f_{0}(s-\tau)\right) d \tau\right\|_{0, l-\sigma, \varrho} \\
& \leqq C \sup _{s \leqq \tau \leqq t}\left\|f_{0}(\tau)\right\|_{l-\sigma, \varrho}\left(\frac{t-s}{s}\right)^{\sigma} \\
& +\int_{0}^{s} e^{-\frac{\tau}{\varepsilon} v_{0}} \frac{\kappa}{\varepsilon}\left\|f_{0}(t-\tau)-f_{0}(s-\tau)\right\| \|_{l-\sigma, \varrho} d \tau \\
& \leqq C\left\{\left(\frac{t-s}{s}\right)^{\sigma}+\int_{0}^{s / 2}+\int_{s / 2}^{s} \frac{e^{-\frac{\tau}{\varepsilon} v_{0}}}{\varepsilon}\left(\frac{t-s}{s-\tau}\right)^{\sigma} d \tau\right\} \\
& \leqq C\left(\frac{t-s}{s}\right)^{\sigma} \text {. }
\end{aligned}
$$

The same is true in the norm $B_{j, l-\sigma, \varrho}$ for $j=1,2, \ldots, m-1$ and in the norm $B_{m-\sigma, l-\sigma, \varrho}$. This completes the proof of Proposition 2.2.

\section{§3. The Solution of the Nonlinear Boltzmann Equation for $\varepsilon \in(0,1]$}

In order to get the solution $f_{\varepsilon}(t)$ of the nonlinear Boltzmann equation for any $\varepsilon \in(0,1]$ in a finite time interval (independent of $\varepsilon$ ) we use the abstract nonlinear Cauchy-Kowalewski theorem in the scales of Banach spaces $S_{l}$ and $S_{m, l}(l \geqq 2$, $m \geqq 3$ ), this theorem has been formulated in [12] and [13]. 
Making use of the linear Boltzmann semigroup $\exp \frac{t}{\varepsilon} B_{\varepsilon}$ in (2.26), we use that the nonlinear Boltzmann equation (2.14) is equivalent to the integral equation

$$
f_{\varepsilon}(t)=e^{\frac{t}{\varepsilon} B_{\varepsilon}} f+\int_{0}^{t} e^{\frac{t-s}{\varepsilon} B_{\varepsilon}} \frac{1}{\varepsilon} v \Gamma\left(f_{\varepsilon}(s), f_{\varepsilon}(s)\right) d s .
$$

Definition. Give $l \geqq 2, m \geqq 3$, and suitably small $a>0$, we denote by $\mathbb{B}$ the Banach space consisting of all continuous function $f(t)$ from $t$ in $B_{m, l, \varrho}$ such that

$$
N_{a}[f] \equiv \sup _{\substack{0 \leqq \varrho^{<} \varrho_{0} \\ 0 \leqq t<a\left(\varrho_{0}-\varrho\right)}}\|f(t)\|_{m, l, \varrho}\left(1-t / a\left(\varrho_{0}-\varrho\right)\right)<+\infty .
$$

$N_{a}[f]$ defines a norm on $\mathbb{B}$.

We seek the solution $f_{\varepsilon}(t)$ of $(3.1)$, for any $\varepsilon \in(0,1]$, in the space $\mathbb{B}$, At the same time, we get an estimate for $f_{\varepsilon}(t)$, uniform for $\varepsilon \in(0,1]$, provided that the initial data

$$
f(0)=f \in B_{m, l, \varrho_{0}} \quad\left(\varrho_{0}>0, l \geqq 2, m \geqq 3\right) .
$$

Theorem 3.1. Consider the initial data $f \in B_{m, l, \varrho_{0}}$ for some $\varrho_{0}>0, l \geqq 2, m \geqq 3$ so that

$$
E[f]=\|\| f \|_{m, l, \varrho_{0}}<+\infty .
$$

Then there exist $E_{0}>0, a>0$ such that for any $f$ with $E[f]<E_{0}$ the Equation (3.1) has the unique solution $f_{\varepsilon}(t)$ for any $\varepsilon \in(0,1]$, any $t \in\left[0, a\left(\varrho_{0}-\varrho\right)\right),\left(\varrho \in\left[0, \varrho_{0}\right)\right.$ fixed $)$. Uniformly for $\varepsilon \in(0,1], f_{\varepsilon}(t)$ satisfies the bound

$$
\left\|f_{\varepsilon}(t)\right\|_{m, l, \varrho} \leqq C E[f]
$$

for any $t, \varrho$ as above.

Consider the function $h(t)$ defined by the quadratic term

$$
h(t)=\int_{0}^{t} e^{\frac{t-s}{\varepsilon} B_{\varepsilon}} \frac{1}{\varepsilon} v \Gamma(f(s), g(s)) d s,
$$

where $f$ and $g$ are any functions satisfying $N_{a}[f], N_{a}[g]<+\infty$. A proof of Theorem 3.1 is based on the following proposition:

Proposition 3.1. Let $f, g$ satisfy $N_{a}[f], N_{a}[g]<+\infty$. Then we have

$$
N_{b}[h] \leqq C R N_{b}[g] \leqq C R N_{a}[g] \text { for any } b<a,
$$

where $R=\sup _{\substack{0 \leqq t<b\left(\varrho_{0}-\varrho\right) \\ 0 \leqq \varrho<\varrho_{0}}}\|f(t)\|_{m, l, \varrho}$.

Proof. Noting that $\left(e_{j}(0), v \Gamma\right)_{L^{2}(v)}=\left(\psi_{j}, v \Gamma\right)=0, j=1, \ldots, 5$, Theorem 2.1 gives the following:

$$
\begin{aligned}
h(t) & =\int_{0}^{t}\left[\frac { 1 } { ( 2 \pi ) ^ { 3 / 2 } } \int _ { | \varepsilon k | < \delta } \left\{\sum_{j=1}^{5} e^{\frac{t-s}{\varepsilon} \alpha_{j}(\varepsilon k)} i k\left(e_{j}^{\prime}(-\theta \varepsilon k),(v \Gamma)^{\wedge}\right) e_{j}(\varepsilon k)\right.\right. \\
& \left.+e^{\frac{t-s}{\varepsilon} A_{\varepsilon k}} \frac{1}{\varepsilon}(v \Gamma)^{\wedge}+e^{-\frac{t-s}{\varepsilon} \beta_{1}} \frac{1}{\varepsilon} \cdot Z_{1}(\varepsilon k, t / \varepsilon)(v \Gamma)^{\wedge}\right\} e^{i k \cdot x} d k \\
& \left.+\int_{|\varepsilon k|>\delta}\left\{e^{\frac{t-s}{\varepsilon} A_{\varepsilon k}} \frac{1}{\varepsilon}(v \Gamma)^{\wedge}+e^{-\frac{t-s}{\varepsilon} \beta_{2}} \frac{1}{\varepsilon} Z_{2}(\varepsilon k, t / \varepsilon)(v \Gamma)^{\wedge}\right\} e^{i k \cdot x} d k\right] d s .
\end{aligned}
$$


Define $\beta_{0}=\min \left(v_{0}, \beta_{1}, \beta_{2}\right)$. Taking the norm in $H_{l, \varrho}$ gives

$$
\begin{aligned}
\|h(t)\|_{l, \varrho} \leqq & \int_{0}^{t}\left[\left\{\int_{|\varepsilon k|<\delta}\left(1+k^{2}\right)^{l} e^{2|k| \varrho} \mid \sum e^{\frac{t-s}{\varepsilon} \alpha_{j}(\varepsilon k)}\right.\right. \\
& \left.\left.\cdot i k\left(e_{j}^{\prime}(-\theta \varepsilon k),(v \Gamma)^{\wedge}\right) e_{j}(\varepsilon k)\right|_{L^{2}(v)} ^{2} d k\right\}^{1 / 2} \\
& +\left\{\int_{|\varepsilon k|>\delta}\left(1+k^{2}\right)^{l} e^{2|k| \varrho} \frac{e^{-2 \frac{t-s}{\varepsilon} \beta_{2}}}{\varepsilon^{2}}\left(1+\left\|Z_{2}\right\| \|^{2}\right)\left|(v \Gamma)^{\wedge}\right|_{L^{2}(v)}^{2} d k\right\}^{1 / 2} \\
& \left.+\left\{\int_{|\varepsilon k|<\delta}\left(1+k^{2}\right)^{l} e^{2|k| \varrho} \frac{e^{-2 \frac{t-s}{\varepsilon} \beta_{1}}}{\varepsilon^{2}}\left(1+\left\|Z_{1}\right\| \|^{2}\right)\left|(v \Gamma)^{\wedge}\right|_{L^{2}(v)}^{2} d k\right\}^{1 / 2}\right] d s \\
\leqq & C \int_{0}^{t}\left[\left(\int\left(1+k^{2}\right)^{l} e^{2|k| \varrho} k^{2}\left|(v \Gamma)^{\wedge}\right|^{2} d k\right)^{1 / 2}+\frac{e^{-\frac{t-s}{\varepsilon} \beta_{0}}}{\varepsilon} \mid\|v \Gamma\|_{l, \varrho}\right] d s \\
\leqq & C \int_{0}^{t} \frac{\|v \Gamma(s) \mid\|_{l, \varrho(s)}}{\varrho(s)-\varrho} d s+\int_{0}^{t} \frac{e^{-\frac{t-s}{\varepsilon} \beta_{0}}}{\varepsilon} \mid\|v \Gamma\|_{l, \varrho} d s
\end{aligned}
$$

for some choice of $\varrho(s) \in\left(\varrho, \varrho_{0}-s / a\right)$; we have also used Lemma 2.1. Using the property of $v \Gamma$ from Lemma 2.2 , we have for $m \geqq 3, l \geqq 2$

$$
\begin{aligned}
\|h(t)\|_{l, \varrho} \leqq & C\left\{\int_{0}^{t} \frac{\|f(s)\|\left\|_{m, l, \varrho(s)} \cdot\right\| g(s)\|\|_{m, l, \varrho(s)}}{\varrho(s)-\varrho} d s\right. \\
& \left.+\int_{0}^{t} \frac{e^{-\frac{t-s}{\varepsilon} \beta_{0}}}{\varepsilon}\|f(s)\|\left\|_{m, l, \varrho} \cdot\right\| g(s) \|_{m, l, \varrho} d s\right\} \\
\leqq & C R\left(\int_{0}^{t} \frac{\|g(s)\|_{m, l, \varrho(s)}}{\varrho(s)-\varrho} d s+\int_{0}^{t} \frac{e^{-\frac{t-s}{\varepsilon} \beta_{0}}}{\varepsilon}\|g(s)\|_{m, l, \varrho} d s\right) .
\end{aligned}
$$

This is valid for $0<t<b\left(\varrho_{0}-\varrho\right), 0 \leqq \varrho<\varrho_{0}$, where $b$ is any number less than $a$ such that $\varrho<\varrho(s)<\varrho_{0}-s / b$. Hence

$$
\|h(t)\|_{l, \varrho} \leqq C R N_{b}[g]\left\{\int_{0}^{t} \frac{d s}{\left(1-s / b\left(\varrho_{0}-\varrho(s)\right)\right)(\varrho(s)-\varrho)}+\int_{0}^{t} \frac{e^{-\frac{t-s}{\varepsilon} \beta_{0}}}{\varepsilon} \frac{d s}{\left(1-s / b\left(\varrho_{0}-\varrho\right)\right)}\right\} .
$$

If we now choose $\varrho(s)=\left(\varrho_{0}-s / b+\varrho\right) / 2$, we have

$$
\begin{aligned}
& \sup _{\substack{0 \leqq \varrho^{<} \varrho_{0} \\
0 \leqq t<b\left(\varrho_{0}-\varrho\right)}}\|h(t)\|_{l, \varrho}\left(1-t / b\left(\varrho_{0}-\varrho\right)\right) \\
& \leqq C R N_{b}[g] \sup _{\substack{0 \leqq 0^{<} \varrho_{0} \\
0 \leqq t<b\left(\varrho_{0}-\varrho\right)}}\left(1-t / b\left(\varrho_{0}-\varrho\right)\right) \\
& \text { - }\left\{\frac{2 b\left(t+b\left(\varrho_{0}-\varrho\right)\right) t}{\left(b\left(\varrho_{0}-\varrho\right)\left(b\left(\varrho_{0}-\varrho\right)-t\right)\right.}+\frac{1}{\beta_{0}} \frac{1}{1-t / b\left(\varrho_{0}-\varrho\right)}\right\} \\
& \leqq C\left(4 b+1 / \beta_{0}\right) R N_{b}[g] \text {. }
\end{aligned}
$$


In order to obtain $N_{b}[h]$ from (3.8) we note that (3.6) is equivalent to

$$
h(t)=\int_{0}^{t} e^{\frac{t-s}{\varepsilon} A_{\varepsilon}} \frac{1}{\varepsilon} v \Gamma(f(s), g(s)) d s+\int_{0}^{t} e^{\frac{t-s}{\varepsilon} A_{\varepsilon}} \frac{K}{\varepsilon} h(s) d s .
$$

It follows from the Definition (2.10) of \|\|$_{m, l, \varrho}$ and the smoothing properties (2.19) of $K$ that for $0 \leqq \varrho<\varrho_{0}$ and $0 \leqq t<b\left(\varrho_{0}-0\right)$

$$
\begin{aligned}
\|h(t)\|_{0, l, \varrho} & =\left\|\int_{0}^{t} e^{\frac{t-s}{\varepsilon} A_{\varepsilon}} \frac{1}{\varepsilon} v \Gamma(f, g) d s+\int_{0}^{t} e^{\frac{t-s}{\varepsilon} A_{\varepsilon}} \frac{K}{\varepsilon} k(s) d s\right\| \|_{0, l, \varrho} \\
& \leqq \sup _{v} \int_{0}^{t} e^{-\frac{t-s}{\varepsilon} v(v)} \frac{v(v)}{\varepsilon}\|\Gamma\|_{0, l, \varrho} d s+\int_{0}^{t} e^{-\frac{t-s}{\varepsilon} v(0)} \frac{\kappa}{\varepsilon}\|h(s)\|_{l, \varrho} d s \\
& \leqq C R \sup _{v} \int_{0}^{t} e^{-\frac{t-s}{\varepsilon} v(v)} \frac{v(v)}{\varepsilon} \frac{N_{b}[g]}{1-s / b\left(\varrho_{0}-\varrho\right)} d s \\
& +C \kappa R N_{b}[g] \int_{0}^{t} \frac{e^{-\frac{t-s}{\varepsilon} v(0)}}{\varepsilon} \frac{d s}{1-s / b\left(\varrho_{0}-\varrho\right)} \\
& \leqq C R N_{b}[g] /\left\{1-t / b\left(\varrho_{0}-\varrho\right)\right\} .
\end{aligned}
$$

Repeating the same argument inductively for $j=1,2, \ldots, m$ in the norm $\|\mid\| \|_{j, l, \varrho}$ gives

$$
\|h(t)\|_{m, l, \varrho} \leqq C R N_{b}[g] /\left\{1-t / b\left(\varrho_{0}-\varrho\right)\right\}
$$

in $0 \leqq \varrho<\varrho_{0}, 0 \leqq t<b\left(\varrho_{0}-\varrho\right)$. This completes the proof of Proposition 3.1.

Now we introduce a successive approximation scheme to solve (3.1).

$$
\begin{aligned}
& f_{0}(t)=e^{\frac{t}{\varepsilon} B_{\varepsilon}} f, \\
& g_{0}(t)=\int_{0}^{t} e^{\frac{t-s}{\varepsilon} B_{\varepsilon}} \frac{1}{\varepsilon} v \Gamma\left(f_{0}(s), f_{0}(s)\right) d s, \\
& f_{1}(t)=g_{0}(t)+f_{0}(t), \\
& g_{1}(t)=\int_{0}^{t} e^{\frac{t-s}{\varepsilon} B_{\varepsilon}} \frac{1}{\varepsilon}\left\{v \Gamma\left(f_{1}(s), g_{0}(s)\right)+v \Gamma\left(g_{0}(s), f_{0}(s)\right)\right\} d s, \\
& \vdots \\
& g_{n}(t)=\int_{0}^{t} e^{\frac{t-s}{\varepsilon} B_{\varepsilon}} \frac{1}{\varepsilon}\left\{v \Gamma\left(f_{n}(s), g_{n-1}(s)\right)+v \Gamma\left(g_{n-1}(s), f_{n-1}(s)\right)\right\} d s, \\
& f_{n+1}(t)=g_{n}(t)+f_{n}(t) \\
&=f_{0}(t)+\int_{0}^{t} e^{\frac{t-s}{\varepsilon} B_{\varepsilon}} \frac{1}{\varepsilon} v \Gamma\left(f_{n}(s), f_{n}(s)\right) d s, \\
& n=1,2,3, \ldots .
\end{aligned}
$$

Since we assumed that $f \in B_{m, l, \varrho_{0}}\left(\varrho_{0}>0, l \geqq 2, m \geqq 3\right.$ fixed) it follows from (2.34) that for any $\varrho \in\left[0, \varrho_{0}\right)$ and any $t \geqq 0$

$$
\left\|f_{0}(t)\right\|_{m, l, \varrho} \leqq C\|f\|_{m, l, \varrho} \leqq C\|f\|_{m, l, \varrho 0} \equiv R_{0} .
$$


To estimate $g_{0}(t)$ in \|\|$_{m, l, \varrho}$ we proceed as in the proof of Proposition 3.1

$$
\begin{aligned}
\left\|g_{0}(t)\right\|_{l, \varrho} \leqq & C \int_{0}^{t} \frac{\left\|v \Gamma\left(f_{0}(s), f_{0}(s)\right)\right\|_{l, \varrho(s)}}{\varrho(s)-\varrho} d s \\
& +C \int_{0}^{t} \frac{e^{-\frac{t-s}{\varepsilon} \beta_{0}}}{\varepsilon} \| v \Gamma\left(f_{0}(s), f_{0}(s) \|_{l, \varrho} d s\right. \\
\leqq & C \sup _{0 \leqq s \leqq t}\left\|v \Gamma\left(f_{0}(s), f_{0}(s)\right)\right\|_{l, \varrho} \cdot\left(\frac{t}{\varrho_{0}-\varrho}+\int_{0}^{t} \frac{e^{-\frac{t-s}{\varepsilon} \beta_{0}}}{\varepsilon} d s\right) \\
\leqq & C\left(a_{0}+1 / \beta_{0}\right)\|f\|_{m, l, \varrho}^{2}
\end{aligned}
$$

for $0 \leqq t \leqq a_{0}\left(\varrho_{0}-\varrho\right), 0 \leqq \varrho<\varrho_{0}$, where $a_{0}$ is any positive number. Thus we get

$$
\left\|g_{0}(t)\right\|_{m, l, \varrho} \leqq C\left(a_{0}+1 / \beta_{0}\right)\|f\|_{m, l, \varrho_{0}}^{2} \leqq C R_{0}^{2} .
$$

Therefore for any $a_{0}>0$ fixed, we arrive at

$$
\mu_{0} \equiv \sup _{\substack{0 \leqq \varrho^{<} \varrho_{0} \\ 0 \leqq t \leqq a_{0}\left(\varrho_{0}-\varrho\right)}}\left\|g_{0}(t)\right\|_{m, l, \varrho} \leqq C R_{0}^{2} .
$$

Then it follows from (3.13) and (3.11) that

$$
\begin{gathered}
\left\|f_{1}(t) \mid\right\|_{m, l, \varrho} \leqq R_{0}+\mu_{0} \\
\text { in } 0 \leqq \varrho<\varrho_{0}, 0 \leqq t \leqq a_{0}\left(\varrho_{0}-\varrho\right) .
\end{gathered}
$$

Define $a_{1}=a_{0}>0$,

$$
a_{n+1}=a_{n}\left(1-1 /(n+1)^{2}\right) \text { for } n=1,2, \ldots
$$

and

$$
N_{n}[g]=N_{a_{n}}[g] \text { for } n=0,1,2, \ldots .
$$

To estimate $g_{1}(t)$ we can use Proposition 3.1. In fact we may assume from (3.12), (3.13), and (3.14) that by taking $R_{0}$ suitably small

$$
\left\|f_{0}(t)\right\|_{m, l, \varrho},\left\|f_{1}(t)\right\|_{m, l, \varrho} \leqq R
$$

for $0 \leqq \varrho<\varrho_{0}, 0 \leqq t \leqq a_{1}\left(\varrho_{0}-\varrho\right)$, where $R>0$ will be chosen small later.

Therefore Proposition 3.1 gives that

$$
\begin{aligned}
\mu_{1} & \equiv N_{1}\left[g_{1}\right] \leqq C R N_{0}\left[g_{0}\right] \leqq C R \sup _{\substack{0 \leqq \varrho^{2} \\
0 \leqq \\
\overline{2} \varrho_{0}\left(\varrho_{0}-\varrho\right)}}\left\|g_{0}(t)\right\| \|_{m, l, \varrho} \\
& =C R \mu_{0}<+\infty .
\end{aligned}
$$

Hence for $0 \leqq \varrho<\varrho_{0}, 0 \leqq t<a_{2}\left(\varrho_{0}-\varrho\right)$

$$
\left\|g_{1}(t)\right\|_{m, l, \varrho} \leqq \mu_{1} /\left(1-a_{2} / a_{1}\right)<+\infty
$$

and this gives for $0 \leqq t<a_{2}\left(\varrho_{0}-\varrho\right)$

$$
\begin{aligned}
\left\|f_{2}(t)\right\|_{m, l, \varrho} & \leqq \frac{\mu_{1}}{1-a_{2} / a_{1}}+\left\|f_{1}(t)\right\|_{m, l, \varrho} \\
& \leqq \frac{\mu_{1}}{1-a_{2} / a_{1}}+\mu_{0}+R_{0} .
\end{aligned}
$$


We will require that for $k=1,2, \ldots$

$$
\sum_{j=1}^{k} \frac{\mu_{j}}{1-a_{j+1} / a_{j}}+\mu_{0}+R_{0}<R .
$$

Then we obtain for $0 \leqq \varrho<\varrho_{0}, 0 \leqq t<a_{k+1}\left(\varrho_{0}-\varrho\right)$

$$
\left\|f_{k+1}(t)\right\|_{m, l, \varrho}<R .
$$

Hence we can construct $g_{k+1}(t)$ in (3.11) and use Proposition 3.1 again to conclude that

$$
\mu_{k+1} \equiv N_{k+1}\left[g_{k+1}\right] \leqq C R \mu_{k} .
$$

Now we choose $R$ such that

$$
\mu_{j} \leqq \mu_{0}(j+1)^{-4} \quad j=1,2, \ldots .
$$

In fact if we assume this is true for $\mu_{k}$, then we find from (3.18) that

$$
\begin{aligned}
\mu_{k+1} & \leqq C R \mu_{k} \leqq C R \mu_{0}(k+1)^{-4} \leqq \mu_{0}(k+2)^{-4}\left\{C R\left(\frac{k+2}{k+1}\right)^{4}\right\} \\
& \leqq \mu_{0}(k+2)^{-4}
\end{aligned}
$$

provided

$$
R<\left(\frac{2}{3}\right)^{4} / C .
$$

Hereafter fix $R$ as in (3.20). At last we can choose $R_{0}$ so small that (3.17) is valid for this choice of $R$. In fact it follows from (3.15), (3.19), and (3.13) that

$$
\begin{aligned}
\sum_{j=1}^{k} \frac{\mu_{j}}{1-a_{j+1} / a_{j}}+\mu_{0}+R_{0} & \leqq \mu_{0} \sum_{j=0}^{+\infty}(j+1)^{-2}+R_{0} \\
& \leqq R_{0}+C R_{0}^{2} \Sigma(j+1)^{-2}<R
\end{aligned}
$$

provided that $R_{0}$ is small. Because of (3.12), this is achieved if $\|f \mid\|_{m, l, \varrho_{0}}$ is small.

Therefore we arrive at the desired estimate for $k=0,1,2, \ldots$

$$
\left\|f_{k+1}(t)\right\|_{m, l, \varrho}<R
$$

for $0 \leqq t<a\left(\varrho_{0}-\varrho\right), 0 \leqq \varrho<\varrho_{0}$, where $a=a_{0} \prod_{j=1}^{\infty}\left(1-(j+1)^{-2}\right)>0$. Furthermore for $k=0,1,2, \ldots, 0 \leqq t<a\left(\varrho_{0}-\varrho\right), 0 \leqq \varrho<\varrho_{0}$

$$
\begin{aligned}
\left\|f_{k+1}(t)-f_{k}(t)\right\|_{m, l, \varrho} & =\left\|g_{k}(t)\right\|_{m, l, \varrho} \\
& \leqq \mu_{k} /\left(1-t / a_{k}\left(\varrho_{0}-\varrho\right)\right) \leqq \mu_{k} /\left(1-t / a\left(\varrho_{0}-\varrho\right)\right) .
\end{aligned}
$$

Thus, we have shown

$$
N_{a}\left[f_{k+1}-f_{k}\right] \leqq \mu_{k} .
$$


Since $\Sigma \mu_{k}<+\infty$, it follows that the $f_{k}$ converge to some $f_{\varepsilon}(t)$ in $\mathbb{B}$ for any $\varepsilon \in(0,1]$ fixed. Also (3.22) gives

$$
\left\|f_{\varepsilon}(t)\right\|_{m, l, \varrho} \leqq R \quad \text { in } \quad 0 \leqq t<a\left(\varrho_{0}-\varrho\right),
$$

where $R$ is independent of $\varepsilon \in(0,1]$.

The limit function $f_{\varepsilon}(t)$ is the desired unique solution of (3.1). This completes the proof of Theorem 3.1.

\section{$\S 4$. Fluid Dynamical Limit as $\varepsilon \rightarrow 0$ to the Level of the Compressible Euler Equation}

In order to take the limit of $f_{\varepsilon}(t)$ as $\varepsilon \rightarrow 0$ we need more than the uniform bound (3.5) for $f_{\varepsilon}(t)$ in $\varepsilon \in(0,1]$. For this purpose we show the Hölder continuity of $f_{\varepsilon}(t)$ in $t$ uniformly for $\varepsilon \in(0,1]$. Let us consider the initial data $f(0) \in B_{m, l, \varrho_{0}}\left(\varrho_{0}>0, l \geqq 2\right.$, $m \geqq 3$ ), for which Theorem 3.1 holds; i.e., if

$$
\|f f(0) \mid\|_{m, l, \varrho_{0}} \equiv E[f]<E_{0},
$$

then the corresponding solution $f_{\varepsilon}(t)$ satisfies the uniform bound

$$
\left\|f_{\varepsilon}(t)\right\|_{m, l, \varrho} \leqq C E[f] \text { for } 0 \leqq t<a\left(\varrho_{0}-\varrho\right), \quad 0 \leqq \varrho<\varrho_{0} .
$$

Definition. For $0<\sigma<1 / 2$ fixed and $l \geqq \sigma, m \geqq 3$,

$$
M_{a}[f] \equiv \sup _{\substack{0<s<t<a\left(\varrho_{0}-\varrho\right) \\ 0 \leqq \varrho<\varrho_{0}}}\|f(t)-f(s)\| \|_{m-\sigma, l-\sigma, \varrho}\left\{\frac{s\left(1-t / a\left(\varrho_{0}-\varrho\right)\right)}{t-s}\right\}^{\sigma} .
$$

Theorem 4.1. Let the initial data $f(0)$ satisfy (4.1). Then there exists $E_{1}>0\left(E_{1} \leqq E_{0}\right)$ such that if $E[f]<E_{1}$, then the solution of (3.1) is Hölder continuous in $t$ :

$$
M_{a}\left[f_{\varepsilon}\right] \leqq C_{1} E,
$$

where $C_{1}$ is independent of $\varepsilon \in(0,1]$.

Before we prove this theorem, we reconsider the quadratic term (3.6):

$$
h(t)=\int_{0}^{t} e^{\frac{t-s}{\varepsilon} B_{\varepsilon}} \frac{1}{\varepsilon} v \Gamma(f(s), g(s)) d s
$$

where $f(s), g(s)$ are any functions which satịfy the bounds

$$
\|\| f\left\|_{m, l, \varrho}, \quad\right\| g \|_{m, l, \varrho}<R,
$$

[for $\left.0 \leqq t<a\left(\varrho_{0}-\varrho\right), 0 \leqq \varrho<\varrho_{0}\right]$ and which are Hölder continuous :

$$
M_{a}[f], M_{a}[g]<+\infty \text {. }
$$

The proof of Theorem 4.1 depends on the following proposition. 
Proposition 4.1. Let us consider $h(t)$ in (4.5) with $f(t), g(t)$ satisfying (4.6) and (4.7). Then for the same $\sigma$ and any numbers $s, t$ satisfying $0<s<t<a\left(\varrho_{0}-\varrho\right)$,

$$
\begin{aligned}
& \|h(t)-h(s)\|_{l-\sigma, \varrho} \leqq C\left(R+M_{a}[g]+M_{a}[f]\right) R\left\{\frac{t-s}{s\left(1-t / a\left(\varrho_{0}-\varrho\right)\right)}\right\}^{\sigma}, \\
& M_{a}[h] \leqq C R\left(R+M_{a}[g]+M_{a}[f]\right),
\end{aligned}
$$

where $C$ is independent of $\varepsilon \in(0,1]$.

Proof of Proposition 4.1. We have

$$
\begin{aligned}
h(t)-h(s)= & \int_{s}^{t} e^{\frac{\tau}{\varepsilon} B_{\varepsilon}} \frac{v}{\varepsilon} \Gamma(f(t-\tau), g(t-\tau)) d \tau \\
& +\int_{0}^{s} e^{\frac{\tau}{\varepsilon} B_{\varepsilon}} \frac{v}{\varepsilon}\{\Gamma(f(t-\tau), g(t-\tau))-\Gamma(f(s-\tau), g(s-\tau))\} d \tau \\
\equiv & I_{1}+I_{2} .
\end{aligned}
$$

By Theorem 2.1 we have

$$
\begin{aligned}
I_{1}= & \int_{s}^{t}\left[\frac { 1 } { ( 2 \pi ) ^ { 3 / 2 } } \int _ { | \varepsilon k | < \delta } e ^ { i k \cdot x } \left\{\sum e^{\frac{\tau}{\varepsilon} \alpha_{j}(\varepsilon k)}\left(e_{j}(-\varepsilon k), \frac{v}{\varepsilon} \hat{\Gamma}(f, g)\right) e_{j}(\varepsilon k)\right.\right. \\
& \left.+e^{-\beta_{1} \frac{\tau}{\varepsilon}} Z_{1}(\varepsilon k, \tau / \varepsilon) \frac{v}{\varepsilon} \hat{\Gamma}(f, g)\right\} d k+\int e^{i k \cdot x} e^{\frac{\tau}{\varepsilon} A_{\varepsilon k}} \frac{v}{\varepsilon} \hat{\Gamma}(f, g) d k \\
& \left.+\frac{1}{(2 \pi)^{3 / 2}} \int_{|\varepsilon k|>\delta} e^{i k \cdot x} e^{-\beta_{2} \frac{\tau}{\varepsilon}} Z_{2}(\varepsilon k, \tau / \varepsilon) \frac{v}{\varepsilon} \hat{\Gamma}(f, g) d k\right] d \tau \\
\equiv & I_{3}+I_{4}+I_{5}+I_{6} .
\end{aligned}
$$

For $I_{3}$, we have

$$
\begin{aligned}
\left\|I_{3}\right\|_{l-\sigma, \varrho} & =\left\|\int_{s}^{t} \frac{1}{(2 \pi)^{3 / 2}} \int_{|\varepsilon k|<\delta} e^{i k \cdot x}\left\{\sum e^{\frac{\tau}{\varepsilon} \alpha_{j}(\varepsilon k)} i k\left(e_{j}^{\prime}(-\theta \varepsilon k), v \hat{\Gamma}\right) e_{j}(\varepsilon k)\right\} d k \mid\right\|_{l-\sigma, \varrho} \\
& \leqq \int_{s}^{t} \frac{C\|v \Gamma(f(t-\tau), g(t-\tau))\|_{l, \varrho(t-\tau)}}{\{\varrho(t-\tau)-\varrho\}^{1-\sigma}} d \tau \\
& =\int_{0}^{t-s} \frac{C\|v \Gamma(f(\tau), g(\tau))\|_{l, \varrho(\tau)}}{\{\varrho(\tau)-\varrho\}^{1-\sigma}} d \tau,
\end{aligned}
$$

where we can set $\varrho(\tau)=\left(\varrho_{0}+\varrho-\tau / a\right) / 2$,

$$
\leqq C R^{2} \int_{0}^{t-s}\left\{\frac{2 a}{a\left(\varrho_{0}-\varrho\right)-\tau}\right\}^{1-\sigma} d \tau \leqq C R^{2}(t-s)^{\sigma} .
$$


The other terms in $I_{1}$ can be treated as follows.

$$
\begin{aligned}
\left\|I_{4}+I_{5}+I_{6}\right\|_{l-\sigma, \varrho} & \leqq C \int_{s}^{t} \frac{1}{\varepsilon} e^{-\beta_{0} \frac{\tau}{\varepsilon}}\|v \Gamma\|_{l-\sigma, \varrho} d \tau \\
& \leqq C\|\Gamma\|_{m-\sigma, l-\sigma, \varrho} \int_{s}^{t} \frac{1}{\varepsilon} e^{-\beta_{0} \frac{\tau}{\varepsilon}} d \tau \leqq C R^{2}\left(\frac{t-s}{s}\right)^{\sigma},
\end{aligned}
$$

where $\beta_{0}=\min \left(v(0), \beta_{1}, \beta_{2}\right)$.

Now we estimate $I_{2}$, noting that if $s / 3<t-s$, then $I_{2}$ may be estimated in the same way as $I_{1}$.

$$
\begin{aligned}
I_{2}= & \int_{0}^{s} e^{\frac{\tau}{\varepsilon} B_{\varepsilon}} \frac{v}{\varepsilon}\{\Gamma(f(t-\tau)-f(s-\tau), g(t-\tau)) \\
& +\Gamma(f(s-\tau), g(t-\tau)-g(s-\tau))\} d \tau .
\end{aligned}
$$

Since each of the two terms in the integrand can be treated analogously, we treat the first term here. We have

$$
\begin{aligned}
& \int_{0}^{s} e^{\frac{\tau}{\varepsilon} B_{\varepsilon}} \frac{v}{\varepsilon} \Gamma(f(t-\tau)-f(s-\tau), g(t-\tau)) d \tau \\
& =\int_{0}^{s} \frac{1}{(2 \pi)^{3 / 2}}\left[\int _ { | \varepsilon k | < \delta } e ^ { i k \cdot x } \left\{\sum e^{\frac{\tau}{\varepsilon} \alpha_{j}(\varepsilon k)}\left(e_{j}(-\varepsilon k), \frac{v}{\varepsilon} \hat{\Gamma}\right) e_{j}(\varepsilon k)\right.\right. \\
& \left.+e^{-\beta_{1} \frac{\tau}{\varepsilon}} Z_{1}(\varepsilon k, \tau / \varepsilon) \frac{v}{\varepsilon} \hat{\Gamma}\right\} d k \\
& \left.+\int e^{i k \cdot x} e^{\frac{\tau}{\varepsilon} A_{\varepsilon k} \nu} \frac{\nu}{\varepsilon} \hat{\Gamma} d k+\int_{|\varepsilon k|>\delta} e^{i k \cdot x} e^{-\beta_{2} \frac{\tau}{\varepsilon}} Z_{2}(\varepsilon k, \tau / \varepsilon) \frac{\nu}{\varepsilon} \hat{\Gamma} d k\right] d \tau \\
& \equiv I_{8}+I_{9}+I_{10}+I_{11} \\
& \left\|I_{8}\right\|_{l-\sigma, \sigma}=\left\|\int_{0}^{s} \frac{1}{(2 \pi)^{3 / 2}} \int_{|\varepsilon k|<\delta} \sum e^{\frac{\tau}{\varepsilon} \alpha_{j}(\varepsilon k)} i k\left(e_{j}^{\prime}, \nu \Gamma\right) e_{j}(\varepsilon k) e^{i k \cdot x} d k\right\| \|_{l-\sigma, \varrho} d \tau \\
& \leqq C \int_{t-s}^{t} \frac{\|v \Gamma(f(\tau)-f(\tau-(t-s)), g(\tau))\|_{l-\sigma, \varrho(\tau)}}{\varrho(\tau)-\varrho} d \tau \\
& \leqq C \int_{t-s}^{t} \frac{\|f(\tau)-f(\tau-(t-s))\|\left\|_{m-\sigma, l-\sigma, \varrho(\tau)} \mid\right\| g(\tau)\|\|_{m-\sigma, l-\sigma, \varrho(\tau)}}{\varrho(\tau)-\varrho} d \tau \\
& \leqq C \int_{t-s}^{t}\left\{\frac{t-s}{(\tau-(t-s))\left(1-\frac{\tau}{\left(\varrho_{0}-\varrho(\tau)\right)}\right)}\right\}^{\sigma} \frac{M_{a}[f] R}{\varrho(\tau)-\varrho} d \tau \\
& =C M_{a}[f] R(t-s)^{\sigma} 2 a \int_{0}^{s} \frac{\left\{a\left(\varrho_{0}-\varrho\right)+\tau+t-s\right\}^{\sigma} d \tau}{\tau^{\sigma}\left\{a\left(\varrho_{0}-\varrho\right)-(t-s+\tau)\right\}^{1+\sigma}} \\
& \leqq C M_{a}[f] R(t-s)^{\sigma} 2 a\left\{a\left(\varrho_{0}-\varrho\right)+t\right\}^{\sigma}\left(\int_{0}^{s / 3}+\int_{s / 3}^{s}\right) \\
& \leqq C M_{a}[f] R\left(\frac{t-s}{s}\right)^{\sigma} \frac{a}{\left(1-t / a\left(\varrho_{0}-\varrho\right)\right)^{\sigma}} .
\end{aligned}
$$


Setting $\beta_{0}=\min \left(v_{0}, \beta_{1}, \beta_{2}\right)$, we estimate $I_{9}, I_{10}$, and $I_{11}$ as follows.

$$
\begin{aligned}
\| I_{9} & +I_{10}+I_{11} \mid \|_{l-\sigma, \varrho} \\
& \leqq C \int_{0}^{s} e^{-\beta_{0} \frac{\tau}{\varepsilon}} \frac{1}{\varepsilon}\|v \Gamma(f(t-\tau)-f(s-\tau), g(t-\tau))\| \|_{l-\sigma, \varrho} d \tau \\
& \leqq C \int_{0}^{s} e^{-\beta_{0} \frac{\tau}{\varepsilon}} \frac{1}{\varepsilon}\|f(t-\tau)-f(s-\tau)\|\left\|_{m-\sigma, l-\sigma, \varrho}\right\| g(t-\tau) \|_{m-\sigma, l-\sigma, \varrho} d \tau \\
& \leqq C R M_{a}[f] \int_{0}^{s} \frac{1}{\varepsilon} e^{-\beta_{0} \frac{\tau}{\varepsilon}}\left(\frac{t-s}{(s-\tau)\left(1-\frac{t-\tau}{a\left(\varrho_{0}-\varrho\right)}\right)}\right)^{\sigma} d \tau \\
& \leqq C R M_{a}[f]\left(\frac{t-s}{1-\frac{t}{a\left(\varrho_{0}-\varrho\right)}}\right)^{\sigma} \int_{0}^{s} \frac{1}{\varepsilon} e^{-\beta_{0} \frac{\tau}{\varepsilon}} \frac{d \tau}{(s-\tau)^{\sigma}} \\
& =C R M_{a}[f]\left(\frac{t-s}{t}\right)^{\sigma}\left(\int_{0}^{s / 2}+\int_{s / 2}^{s}\right) \\
& \leqq
\end{aligned}
$$

Thus the desired estimate (4.8) is obtained. Now the procedure for obtaining (4.9) from (4.8) is analogous to that used in Proposition 2.2; i.e., we use the expression (3.9), Lemma 2.4, and the argument for (2.42) with the smoothing properties of $K$ (2.19).

This completes the proof of Proposition 4.1.

Proof of Theorem 4.1. Since the initial data $f \in B_{m, l, \varrho_{0}}\left(\varrho_{0}>0, l \geqq 2, m \geqq 3\right)$ satisfies (4.1), the solution $f_{\varepsilon}(t)$ of (2.14) exists and satisfies $\left\|f_{\varepsilon}(t)\right\|_{m, l, \varrho} \leqq C E$ for any $\varepsilon \in(0,1]$. Also

$$
f_{\varepsilon}(t)=e^{\frac{t}{\varepsilon} B_{\varepsilon}} f+\int_{0}^{t} e^{\frac{t-s}{\varepsilon} B_{\varepsilon}} \frac{\nu}{\varepsilon} \Gamma\left(f_{\varepsilon}(s), f_{\varepsilon}(s)\right) d s .
$$

Remembering the definition of $M_{a}\left[f_{\varepsilon}\right]$ in (4.3) and using Proposition 2.2 and Proposition 4.1 we have the following for $l>\sigma, m \geqq 3$ :

$$
\begin{aligned}
M_{a}\left[f_{\varepsilon}\right] \leqq & \sup _{\substack{0<s<t<a\left(\varrho_{0}-\varrho\right) \\
0 \leqq \varrho<\varrho_{0}}} C\|f\| \|_{m, l, \varrho}\left(1-t / a\left(\varrho_{0}-\varrho\right)\right)^{\sigma} \\
& +C E\left(E+M_{a}\left[f_{\varepsilon}\right]\right) \leqq C_{1} E+C E M_{a}\left[f_{\varepsilon}\right] .
\end{aligned}
$$

Therefore if $C E<1$, then

$$
M_{a}\left[f_{\varepsilon}\right] \leqq \frac{C_{1} E}{1-C E},
$$

where $C, C_{1}, E$ is independent of $\varepsilon \in(0,1]$. 
This completes the proof.

From Theorems 3.1 and 4.1 we conclude the following: the solution $f_{\varepsilon}(t)$ is bounded in the norm \|\|$\|_{m, l, \varrho}$ for $0 \leqq t<a\left(\varrho_{0}-\varrho\right)\left(0 \leqq \varrho<\varrho_{0}\right)$, uniformly for $\varepsilon \in(0,1]$; in the space $B_{m-\sigma, l-\sigma, e}, f_{\varepsilon}(t)$ is $\sigma$-Hölder continuous in $t$, uniformly for $\varepsilon \in(0,1]$, for $t$ in any compact subset of the interval $\left(0, a\left(\varrho_{0}-\varrho\right)\right)\left[\right.$ where $\left.\varrho \in\left[0, \varrho_{0}\right)\right]$. Therefore by the Ascoli-Arzela lemma we can choose a convergent subsequence $\left\{f_{\varepsilon_{j}}\right\}$, where $\varepsilon_{j} \rightarrow 0$, such that

$$
f_{\varepsilon_{j}}(t) \rightarrow f_{0}(t), \text { for } 0<t<a\left(\varrho_{0}-\varrho\right), \quad 0 \leqq \varrho<\varrho_{0} .
$$

The limit function satisfies the bound

$$
\left\|f_{0}(t)\right\|_{m, l, \varrho} \leqq C E \text { in } \quad 0 \leqq t<a\left(\varrho_{0}-\varrho\right), \quad 0 \leqq \varrho<\varrho_{0},
$$

and is $\sigma$-Hölder continuous $(0<\sigma<1 / 2)$ :

$$
\left\|f_{0}(t)-f_{0}(s)\right\|_{m-\sigma, l-\sigma, \varrho} \leqq C\left(\frac{t-s}{s\left(1-t / a\left(\varrho_{0}-\varrho\right)\right)}\right)^{\sigma} .
$$

Now we turn to the original mass density distribution function

$$
F_{\varepsilon}(t, x, v)=M(v)+M(v)^{1 / 2} f_{\varepsilon}(t, x, v),
$$

where $M(v)$ is the absolute Maxwellian state. $F_{\varepsilon}(t)$ satisfies the original Boltzmann equation

$$
\frac{\partial F_{\varepsilon}}{\partial t}+\sum_{j=1}^{3} v_{j} \frac{\partial F_{\varepsilon}}{\partial x_{j}}=\frac{1}{\varepsilon} Q\left(F_{\varepsilon}, F_{\varepsilon}\right) .
$$

Integration with respect to $t$ gives

$$
\begin{aligned}
& F_{\varepsilon}(t, x, v)-F_{\varepsilon}(0, x, v)+\int_{0}^{t} \sum_{j=1}^{3} v_{j} \frac{\partial F_{\varepsilon}(s, x, v)}{\partial x_{j}} d s \\
& \quad=\frac{1}{\varepsilon} \int_{0}^{t} Q\left(F_{\varepsilon}(s), F_{\varepsilon}(s)\right) d s \text { for } 0<t<a\left(\varrho_{0}-\varrho\right), \quad 0 \leqq \varrho<\varrho_{0} .
\end{aligned}
$$

Noting the uniform bound (4.2) on $f_{\varepsilon}(t)$ for $\varepsilon \in(0,1]$ and the convergence (4.10) along the subsequence $\varepsilon_{j} \rightarrow 0$, we can conclude from (4.14) that

$$
\int_{0}^{t} Q\left(F_{0}(s), F_{0}(s)\right) d s=0
$$

for any $t$ satisfying $0<t<a\left(\varrho_{0}-0\right)\left(0 \leqq \varrho<\varrho_{0}\right)$, where $F_{0}(t, x, v)=M(v)$ $+M(v)^{1 / 2} f_{0}(t, x, v)$.

We now state the main theorem.

Theorem. Assume that the initial data $F(x, v) \equiv M+M^{1 / 2} f(x, v) \geqq 0$, that $\varrho(0, x)$ $=\int F(x, v) d v>0$ for any $x \in R^{3}$, and that $f(x, v) \in B_{m, l, e_{0}}$ for some $\varrho_{0}>0, l \geqq 2, m \geqq 3$; set $\|f\|_{m, l, e_{0}}=E$ and let $a$ be as in Theorem 3.1. If $E<E_{1}$, where $E_{1}$ is defined in Theorem 4.1, then for any $\varepsilon \in(0,1]$ the solution $F_{\varepsilon}(t, x, v)$ of the nonlinear Boltzmann equation (1.1) with the initial data $F(x, v)$ exists in $B_{m, l, \varrho}$ for $0 \leqq t<a\left(\varrho_{0}-\varrho\right) \quad\left(0 \leqq \varrho<\varrho_{0}\right)$ and is nonnegative there. Furthermore the limit 
$\lim _{\varepsilon \rightarrow 0} F_{\varepsilon}(t, x, v) \equiv F_{0}(t, x, v)$ exists in $B_{m, l, \varrho}$ for $0<t<a\left(\varrho_{0}-\varrho\right)\left(0<\varrho<\varrho_{0}\right)$ and $F_{0}(t, x, v)$ is locally Maxwellian. Hence its hydrodynamical quantities satisfy the nonlinear hyperbolic conservation laws (1.13).

Proof. The existence of $F_{\varepsilon}(t)$ and of the limit $F_{0}(t)$ along a subsequence as $\varepsilon \rightarrow 0$ is proved above. At the same time for each $\varepsilon \in(0,1]$ the conservation laws $(1.10)$ are satisfied by the hydrodynamical quantities $(1.5) \sim(1.9)$ of $F_{\varepsilon}(t, x, v)$. They can be integrated in $t$

$$
\begin{aligned}
& \varrho_{\varepsilon}(t, x)-\varrho_{\varepsilon}(0, x)+\sum_{j} \int_{0}^{t} \frac{\partial \varrho_{\varepsilon} u_{\varepsilon j}}{\partial x_{j}}=0, \\
& \left(\varrho_{\varepsilon} u_{\varepsilon i}\right)(t, x)-\left(\varrho_{\varepsilon} u_{\varepsilon i}\right)(0, x)+\sum_{j} \int_{0}^{t} \frac{\partial\left(\varrho_{\varepsilon} u_{\varepsilon i} u_{\varepsilon j}+p_{\varepsilon i j}+p_{\varepsilon} \delta_{i j}\right)}{\partial x_{j}}=0 \\
& \varrho_{\varepsilon}\left(e_{\varepsilon}+\frac{1}{2} u_{\varepsilon}^{2}\right)(t, x)-\varrho_{\varepsilon}\left(e_{\varepsilon}+\frac{1}{2} u_{\varepsilon}^{2}\right)(0, x) \\
& \quad+\sum_{j} \int_{0}^{t} \frac{\partial}{\partial x_{j}}\left\{\varrho_{\varepsilon} u_{\varepsilon j}\left(e_{\varepsilon}+\frac{1}{2} u_{\varepsilon}^{2}\right)+u_{\varepsilon k}\left(p_{\varepsilon k j}+p_{\varepsilon} \delta_{k j}\right)+q_{\varepsilon j}\right\} d s=0,
\end{aligned}
$$

where the initial data for the hydrodynamical quantities $\varrho_{\varepsilon}, u_{\varepsilon}, e_{\varepsilon}$ are given by (1.5) (1.6), and (1.9) with $F=F(x, v)$ and are independent of $\varepsilon \in(0,1]$. It follows from the mass conservation law for $F_{\varepsilon}(t, x, v)\left(\partial \varrho_{\varepsilon} / \partial t+\partial \varrho_{\varepsilon} u_{\varepsilon j} / \partial x_{j}=0\right)$ that $\varrho_{\varepsilon}(0, x)=\varrho(0, x)>0$ implies $\varrho_{\varepsilon}(t, x)>0$ for $0 \leqq t<a\left(\varrho_{0}-\varrho\right), 0 \leqq \varrho<\varrho_{0}$, and any $\varepsilon \in(0,1]$. The nonnegativity of $F_{\varepsilon}(t, x, v)$ follows from that of $F_{\varepsilon}(0, x, v)=F(x, v) \geqq 0$ for fixed $\varepsilon>0$ (proved in the Appendix). The convergence of $F_{\varepsilon}$ to $F_{0}$ as $\varepsilon \rightarrow 0$ gives (4.16) with $\varepsilon=0$ for the hydrodynamical quantities $\varrho, u, e$ of $F_{0}$. Since $F_{0}(t, x, v) \geqq 0$ and $\varrho(t, x)>0$, it follows from (4.15) [i.e., $\left.Q\left(F_{0}(t, x, v), F_{0}(t, x, v)\right)=0\right]$ that $F_{0}(t, x, v)>0$ and so $F_{0}(t, x, v)$ is locally Maxwellian. When $F_{0}(t, x, v)$ is locally Maxwellian, we conclude that $p_{i j}=q_{j}=0$. Therefore (4.16) with $\varepsilon=0$ simplifies to

$$
\left.\begin{array}{l}
\varrho(t, x)-\varrho(0, x)=-\sum_{j} \int_{0}^{t} \frac{\partial \varrho u_{j}}{\partial x_{j}} d s \\
\varrho u_{i}(t, x)-\varrho u_{i}(0, x)=-\sum_{j} \int_{0}^{t} \frac{\partial\left(\varrho u_{i} u_{j}+p \delta_{i j}\right)}{\partial x_{j}} d s \\
\varrho\left(e+\frac{1}{2} u^{2}\right)(t, x)-\varrho\left(e+\frac{1}{2} u^{2}\right)(0, x)=-\sum_{j} \int_{0}^{t} \frac{\partial}{\partial x_{j}}\left(\varrho u_{j}\left(e+\frac{1}{2} u^{2}\right)+u_{j} p\right) d s .
\end{array}\right\}
$$

Now the bounds (4.11) on $f_{0}(t)$ carry over to $F_{0}(t)$. Hence the terms on the right hand side of (4.17) are differentiable in $t$ for $0 \leqq t<a\left(\varrho_{0}-\varrho\right)\left(0 \leqq \varrho<\varrho_{0}\right)$. Thus differentiating (4.17) in $t$ gives the desired nonlinear hyperbolic conservation laws (1.13), where the initial data are given by (1.5), (1.6), and (1.9) with $F_{0}(0, x, v)$ $=F(x, v)$. It follows from the uniqueness of the solution of the initial value problem for (1.13) that all sequences of $F_{\varepsilon}$ as $\varepsilon \rightarrow 0$ give rise to the same system (1.13) in the limit.

This completes the proof of the theorem.

Finally we note that the hyperbolic system (1.13), supplemented by (1.11), has two genuine nonlinear characteristic fields in the sense of Lax. Hence in general 
shocks will form in finite time even for the analytic initial data. Thus another detailed considerations are necessary to get the fluid dynamical limit globally in time at the level of the compressible Euler equation or at the level of the compressible Navier-Stokes equation.

\section{Appendix. Non-Negativity of the Solution of Boltzmann Equation}

The unknown function $F(t, x, v)$ of the Boltzmann equation denotes the mass density distribution function of the gas and for physical reasons should be nonnegative whenever the initial data is non-negative. But it seems that the nonnegativity is not proved explicitly either for Grad's local solution [7] in a neighborhood of the absolute Maxwellian state or for the global solution [18, 19, 14]. Here we prove it in the following form:

$$
\begin{aligned}
& \frac{\partial F_{\varepsilon}(t)}{\partial t}=-\sum v_{j} \frac{\partial F_{\varepsilon}(t)}{\partial x_{j}}+\frac{1}{\varepsilon} Q\left(F_{\varepsilon}(t), F_{\varepsilon}(t)\right) \text { in } t \geqq 0, \\
& F_{\varepsilon}(0)=F(x, v) \geqq 0 .
\end{aligned}
$$

Theorem A.1. Let $F(x, v)=M(v)+M(v)^{1 / 2} f(x, v) \geqq 0$, and $f(x, v) \in B_{m, l}$ for some $m \geqq 3, l \geqq 2$. Then there exist two constants $E_{0}>0$ and $t_{0}>0$ such that if $\|f\|_{m, l}<E_{0}$, then there exists a unique non-negative solution to (A.1), (A.2) in $0 \leqq t \leqq \varepsilon t_{0}$.

The solution is obtained by an iteration which preserves the non-negativity.

$$
\begin{aligned}
& F_{0}(t)=F(x, v) \geqq 0, \\
& \frac{\partial F_{n+1}}{\partial t}+v \cdot \frac{\partial F_{n+1}}{\partial x}=\frac{1}{\varepsilon} \int\left(F_{n}^{\prime} F_{n, *}^{\prime}-F_{n, *} F_{n+1}\right) d \Omega \quad n=0,1,2, \ldots,
\end{aligned}
$$

where $d \Omega=V r d r d \phi d v_{*}, F_{n}^{\prime}=F_{n}\left(t, x, v^{\prime}\right)$ and so on. If we substitute $F_{n}(t, x, v)=M(v)$ $+M(v)^{1 / 2} f_{n}(t, x, v)$ into (A.4), we get

$$
\begin{aligned}
& \frac{\partial f_{n+1}(t)}{\partial t}+\frac{1}{\varepsilon} A_{\varepsilon} f_{n+1}(t)+\frac{v}{\varepsilon} \Gamma_{1}\left(f_{n}(t), f_{n+1}(t)\right) \\
& \quad=\frac{1}{\varepsilon}\left\{K f_{n}(t)+v \Gamma_{2}\left(f_{n}(t), f_{n}(t)\right)\right\} \\
& f_{n+1}(0)=f, \quad n=0,1,2, \ldots, \\
& f_{0}(t)=f
\end{aligned}
$$

where $v(v), K$ is the same as (2.18) and (2.19) and

$$
\left.\begin{array}{l}
v \Gamma_{1}(g, f)=\int g_{*} M\left(v_{*}\right)^{1 / 2} d \Omega \cdot f \\
v \Gamma_{2}(g, f)=\frac{1}{2} \int\left(f^{\prime} g_{*}^{\prime}+f_{*}^{\prime} g^{\prime}\right) M\left(v_{*}\right)^{1 / 2} d \Omega .
\end{array}\right\}
$$

The latter may be estimated as in Lemma 2.2 (cf., [7]). Thus our aim is to prove the convergence of this iteration scheme. It is based on the following proposition. 
Proposition A.1. Consider the initial value problem

$$
\begin{aligned}
\frac{\partial f(t)}{\partial t} & +\frac{1}{\varepsilon} A_{\varepsilon} f(t)+\frac{v}{\varepsilon} \Gamma_{1}\left(g(t), f_{n}(t)\right) \\
& =\frac{1}{\varepsilon}\left\{K g(t)+v \Gamma_{2}(g(t), h(t))\right\}, \\
f(0) & =f(x, v) \in B_{m, l}, m \geqq 2, l \geqq 2 .
\end{aligned}
$$

Let $g(t)$ and $h(t)$ be continuous functions in $t \in[0, T]$ with values in $B_{m, l}(m, l \geqq 2)$, and set

$$
G=\max \left(\sup _{0 \leqq t \leqq T}\|g(t)\|_{m, l}, \sup _{0 \leqq t \leqq T}\|h(t)\|_{m, l}\right) .
$$

Then there exists a constant $0<C<\infty$ such that if $C G<1$, (A.7) has a unique solution $f(t)$ which is continuous for $t \in[0, T]$ with values in $B_{m, l}$ and satisfies the estimates:

$$
\|f(t)\|_{m, l} \leqq \frac{\|f\|_{m, l}+G\left(\frac{\kappa}{\varepsilon} t+C G\right)}{1-C G} .
$$

Proof. The solution of (A.7) is given by the iteration

$$
\begin{gathered}
\frac{\partial f_{n+1}(t)}{\partial t}+\frac{1}{\varepsilon} A_{\varepsilon} f_{n+1}(t)=\frac{1}{\varepsilon}\left\{K g(t)+v \Gamma_{2}(g(t), h(t))\right. \\
\left.-v \Gamma_{1}\left(g(t), f_{n}(t)\right)\right\} \\
f_{n+1}(0)=f_{0}(t)=f \in B_{m, l}, \quad n=0,1,2, \ldots .
\end{gathered}
$$

Since $g(t)$ and $h(t)$ are continuous functions of $t$ for $t \in[0, T]$ with values in $B_{m, l}(m, l \geqq 2)$, we see that $K g, \Gamma_{1}(g, h)$ and $\Gamma_{2}(g, h)$ are continuous functions of $t \in[0, T]$ in $B_{m, l}$. The latter satisfy the estimates

$$
\left.\begin{array}{l}
\|K g\|_{m, l} \leqq K\left\|_{g}\right\|_{m, l} \\
\left\|\Gamma_{2}(g, h)\right\|_{m, l} \leqq C\left\|_{g, l}\right\| h \|_{m, l}, \\
\left\|\Gamma_{1}(g, h)\right\|_{m, l} \leqq C\left\|_{g}\right\|_{m, l}\|h\|_{m, l} .
\end{array}\right\}
$$

Equations (A.10) are equivalent to

$$
\left.\begin{array}{rl}
f_{n+1}(t) & =e^{\frac{t}{\varepsilon} A_{\varepsilon}} f+\int_{0}^{t} e^{\frac{t-s}{\varepsilon} A_{\varepsilon}} \frac{1}{\varepsilon}\left(K g+v \Gamma_{2}(g, h)\right. \\
& \left.-v \Gamma_{1}\left(g, f_{n}\right)\right) d s, \quad n=0,1,2, \ldots
\end{array}\right\}
$$


Thus we have for $C G<1$

$$
\begin{aligned}
\sup _{0 \leqq s \leqq t}\left\|f_{0}(s)\right\|_{m, l} \leqq\|f\|_{m, l} \\
\sup _{0 \leqq s \leqq t}\left\|f_{1}(s)\right\|_{m, l} \leqq\|f\|_{m, l}+\frac{\kappa}{\varepsilon} G t+C G^{2}+C G\|f\|_{m, l} \\
\\
\leqq \frac{\|f\|_{m, l}+G\left(\frac{\kappa}{\varepsilon} t+C G\right)}{1-C G}
\end{aligned}
$$

For $j=0,1,2, \ldots, n$, let

$$
\sup _{0 \leqq s \leqq t}\left\|f_{j}(s)\right\|_{m, l} \leqq \frac{\|f\|_{m, l}+G\left(\frac{\kappa}{\varepsilon} t+C G\right)}{1-C G}
$$

Then by (A.12)

$$
\begin{aligned}
\sup _{0 \leqq s \leqq t}\left\|f_{n+1}(s)\right\|_{m, l} & \leqq\|f\|_{m, l}+\frac{\kappa}{\varepsilon} G t+C G^{2}+C G \sup _{0 \leqq s \leqq t}\left\|f_{n}(s)\right\|_{m, l} \\
& \leqq \frac{\|f\|_{m, l}+G\left(\frac{\kappa}{\varepsilon} t+C G\right)}{1-C G} \quad(n=0,1,2, \ldots) .
\end{aligned}
$$

Also by (A.12)

$$
\begin{aligned}
\sup _{0 \leqq s \leqq t}\left\|f_{n+1}(s)-f_{n}(s)\right\|_{m, l} & \leqq C G \sup _{0 \leqq s \leqq t}\left\|f_{n}(s)-f_{n-1}(s)\right\|_{m, l} \\
& \leqq(C G)^{n} \sup _{0 \leqq s \leqq t}\left\|f_{1}(s)-f_{0}(s)\right\|_{m, l} \\
& \leqq(C G)^{n}\left\{(2+C G)\|f\|_{m, l}+G\left(\frac{\kappa}{\varepsilon} t+C G\right)\right\} .
\end{aligned}
$$

Therefore the iteration (A.10) converges to the solution of (A.7) and the estimate (A.9) is a consequence of (A.13). This completes the proof of Proposition A.1.

Now we return to the iteration (A.5) and the proof of the theorem. Let $C$ denote the constant in (A.11). Provided that $\sup _{0 \leqq s \leqq t}\left\|f_{n}(s)\right\|_{m, l}<1 / C$, the equations in (A.5) may be solved successively for $f_{n+1}$ in terms of $f_{n}$ because of Proposition A.1. In fact, we prove that if the initial data is so small that

$$
\|f\|_{m, l} \leqq 1 / 24 C,
$$

then

$$
G_{n}=\max _{j=0,1, \ldots, n} \sup _{0 \leqq s \leqq t}\left\|f_{j}(s)\right\|_{m, l} \leqq 4\|f\|_{m, l} \leqq 1 / 6 C, \quad n=0,1,2, \ldots .
$$


By Proposition A.1 with $g=f_{0}(t)=f$ and by (A.14), we have

$$
\begin{aligned}
\sup _{0 \leqq s \leqq t}\left\|f_{1}(s)\right\|_{m, l} & \leqq \frac{\|f\|_{m, l}+\|f\|_{m, l}\left(\frac{\kappa}{\varepsilon} t+C\|f\|_{m, l}\right)}{1-C\|f\|_{m, l}} \\
& \leqq 2\|f\|_{m, l},
\end{aligned}
$$

provided that

$$
\frac{\kappa}{\varepsilon} t \leqq 1 / 4
$$

Then for $t \leqq \varepsilon / 4 \kappa$, we have

$$
\sup _{0 \leqq s \leqq t}\left\|f_{1}(s)-f_{0}(s)\right\|_{m, l} \leqq 3\|f\|_{m, l} .
$$

Let (A.15) be true for $j=0,1, \ldots, n$, as the hypothesis of induction. Then by Proposition A.1 we have

$$
\begin{aligned}
\sup _{0 \leqq s \leqq t}\left\|f_{n+1}(s)\right\|_{m, l} & \leqq \frac{\|f\|_{m, l}+G_{n}\left(\frac{\kappa}{\varepsilon} t+C G_{n}\right)}{1-C G_{n}} \\
& \leqq \frac{16}{5}\|f\|_{m, l} \leqq 1 / 6 C,
\end{aligned}
$$

where (A.15) is used. Thus $f_{n+1}(t)$ is well-defined for $n=0,1, \ldots$ and satisfies (A.15). Also the difference $h_{n}(t)=f_{n+1}(t)-f_{n}(t)$ satisfies the equation

$$
\begin{aligned}
\frac{\partial h_{n}(t)}{\partial t}+ & \frac{1}{\varepsilon} A_{\varepsilon} h_{n}(t)+\frac{1}{\varepsilon}\left(v \Gamma_{1}\left(f_{n}(t), h_{n}(t)\right)\right. \\
= & \frac{1}{\varepsilon}\left\{K h_{n-1}(t)+v \Gamma_{2}\left(f_{n}(t), h_{n-1}(t)\right)\right. \\
& \left.+v \Gamma_{2}\left(h_{n-1}(t), f_{n-1}(t)\right)-v \Gamma_{1}\left(h_{n-1}(t), f_{n}(t)\right)\right\}, \\
h_{n}(0)= & 0 .
\end{aligned}
$$

It follows from (A.15) and Proposition A.1 that the following estimate holds for $\frac{\kappa}{\varepsilon} t \leqq 1 / 4$ :

$$
\begin{aligned}
\sup _{0 \leqq s \leqq t}\left\|h_{n}(s)\right\|_{m, l} & \leqq \frac{\left(\frac{\kappa}{\varepsilon} t+3 C G\right) \sup _{0 \leqq s \leqq t}\left\|h_{n-1}(s)\right\|_{m, l}}{1-C G} \\
& \leqq\left(\frac{9}{10}\right)_{0 \leqq s \leqq t}^{n} \sup _{0}\left\|h_{0}(s)\right\|_{m, l} \leqq\left(\frac{9}{10}\right)^{n} 3\|f\|_{m, l} .
\end{aligned}
$$

Therefore as $n \rightarrow+\infty f_{n}(t)$ converges to $f(t)$ in $B_{m, l}, 0 \leqq t \leqq \frac{\varepsilon}{4 \kappa}$, which gives the solution of Boltzmann equation. This completes the proof of Theorem A.1. 
$N . B$. Since the uniqueness of the solution of the initial value problem for the Boltzmann equation is known in a neighborhood of the absolute Maxwellian state (cf. a remark of Shizuta [17]), the solution we obtain coincides with Grad's solution [7].

Acknowledgement. The author would like to thank Professor Yoshio Sone and Professor Seiji Ukai for discussions on the subject.

\section{References}

1. Chapman,S., Cowling,T.: The mathematical theory of non-uniform gases, 3rd ed. London: Cambridge University Press 1970

2. Ellis, R., Pinsky,M.: The first and second fluid approximations to the linearized Boltzmann equation. J. Math. Pures Appl. 54, 125-156 (1975)

3. Ellis, R., Pinsky,M.: The projection of the Navier-Stokes equations upon the Euler equations. J. Math. Pures Appl. 54, 157-181 (1975)

4. Grad,H.: Principles of the kinetic theory of gases. In: Handbuch der Physik, Vol.12. BerlinGöttingen-Heidelberg: Springer 1958

5. Grad,H.: Asymptotic theory of the Boltzmann equation. Phys. Fluids 6, 147-181 (1963)

6. Grad,H.: Asymptotic theory of the Boltzmann equation. II. In: Rarefied gas dynamics, Vol. 1 (ed. J.Laurmann), pp. 26-59. New York: Academic Press 1963

7. Grad,H.: Asymptotic equivalence of the Navier-Stokes and non-linear Boltzmann equations. Proc. Symp. Appl. Math., Am. Math. Soc. 17, 154-183 (1965)

8. Grad,H.: On Boltzmann's H-theorem. J. Soc. Industr. Appl. Math. 13, 259-277 (1965)

9. Inoue, K., Nishida, T.: On the Broadwell model of the Boltzmann equation for a simple discrete velocity gas. Appl. Math. Optimiz. (Intern. J.) 3, 24-49 (1977)

10. McLennan,J.: Convergence of the Chapman-Enskog expansion for the linearized Boltzmann equation. Phys. Fluids 8, 1580-1584 (1965)

11. Nalimov, V.: A priori estimates of solutions of elliptic equations in the class of analytic functions and their applications to the Cauchy-Poisson problem. Dokl. Akad. Nauk SSSR 189 (1969); English translation: Sov. Math. Dokl. 10, 1350-1354 (1969)

12. Nirenberg,L.: An abstract form of the nonlinear Cauchy-Kowalewski theorem. J. Diff. Geometry 6, 561-576 (1972)

13. Nishida,T.: On the Nirenberg's abstract form of the nonlinear Cauchy-Kowalewski theorem. J. Diff. Geometry (to appear)

14. Nishida,T., Imai,K.: Global solutions to the initial value problem for the nonlinear Boltzmann equation. Publ. Res. Inst. Math. Sci., Kyoto Univ. 12, 229-239 (1976)

15. Ovsjannikov,L.: A nonlinear Cauchy problem in a scale of Banach spaces. Dokl. Akad. Nauk SSSR 200 (1971); English translation: Sov. Math. Dokl. 12, 1497-1502 (1971)

16. Pinsky, M.: On the Navier-Stokes approximation to the linearized Boltzmann equation. J. Math. Pures Appl. 55, 217-231 (1976)

17. Shizuta, Y.: The existence and approach to equilibrium of classical solutions of the Boltzmann equation. Commun. math. Phys. (to appear)

18. Ukai,S.: On the existence of global solutions of mixed problem for nonlinear Boltzmann equation. Proc. Japan Acad. 50, 179-184 (1974)

19. Ukai, S.: Les solutions globales de l'équation nonlinéaire de Boltzmann dans l'espace tout entier et dans le demi-espace. Compte Rendu Acad. Sci. Paris 282 A, 317-320 (1976)

20. Ukai,S., Nishida,T.: On the Boltzmann equation (Proc. Coll. Franco-Japan 1976). To appear

Communicated by J. Glimm

Received September 28, 1977; in revised form March 31, 1978 